\title{
Composite chiral structures for morphing airfoils: Numerical analyses and development of a manufacturing process
}

\author{
Paolo Bettini ${ }^{a}$, Alessandro Airoldi ${ }^{\mathrm{a}, *}$, Giuseppe Sala ${ }^{\mathrm{a}}$, Luca Di Landro ${ }^{\mathrm{a}}$, Massimo Ruzzene ${ }^{\mathrm{b}}$, \\ Alessandro Spadoni ${ }^{\mathrm{c}}$ \\ a Dipartimento di Ingegneria Aerospaziale, Politecnico di Milano, Milano, Italy \\ ${ }^{\mathrm{b}}$ School of Aerospace Engineering, Georgia Institute of Technology, Atlanta, GA 30332, USA \\ ${ }^{\mathrm{c}}$ Graduate Aeronautics Laboratories, California Institute of Technology, Pasadena, CA 91125, USA
}

\section{A R T I C L E I N F O}

\section{Article history:}

Received 12 March 2009

Received in revised form 22 September 2009

Accepted 29 September 2009

Available online xxxx

\section{Keywords:}

Morphing structures

E. Assembly

B. Mechanical properties

C. Finite element analysis

\begin{abstract}
A B S T R A C T
This paper investigates complex composite cellular structures featuring a chiral topology with the ability to undergo large overall displacements with limited straining of its components. Numerical analyses are performed to exploit such properties in the design of a morphing airfoil. The advantages associated with the use of composites are investigated by comparing the numerical results with those obtained with a metallic structure. A manufacturing process is then developed to assemble the considered structural configurations using composite materials. The novel process developed is described as applied to the manufacturing of macro chiral components that are tested to evaluate their ability to undergo large deformations. Finally, detailed numerical models of the manufactured structures are presented as a possible approach to evaluate the strength of the proposed configuration.
\end{abstract}

๑ 2009 Elsevier Ltd. All rights reserved.

\section{Introduction}

In the aerospace field, research is currently being conducted to study aircraft structures with morphing capabilities [1]. Morphing structures can expand the flight envelope of a vehicle, eliminate flap-type mechanisms, reduce aerodynamic drag and help control vibrations. A typical objective of a morphing concept is to provide airfoils with continuous deformations to obtain a potentially superior alternative to conventional control surfaces [1,2]. In this sense, a variety of solutions have been proposed to provide aircraft wings and helicopter rotor blades with adaptive capabilities [2,3]. Composite materials can play a fundamental role in the development of such innovative structures, thanks to the wide range of mechanical properties that can be obtained by exploiting material orthotropy and different types of reinforcement. Additionally, new manufacturing processes are expected to improve the effectiveness of innovative structural concepts by possibly embedding micro actuators and sensors for the development of smart morphing structures [4].

This paper investigates the morphing properties of a novel chiral composite structural network that can be manufactured by assembling thin carbon/epoxy or glass/epoxy composite laminates. Chiral

\footnotetext{
* Corresponding author. Address: Dipartimento di Ingegneria Aerospaziale, Politecnico di Milano, Via La Masa, 34, 20156 Milano, Italy. Tel.: +39 0223998363; fax: +39 0223998334.

E-mail address: alessandro.airoldi@polimi.it (A. Airoldi).
}

topologies present a deformation mechanism that inherently opposes unit-cell shape variation [5,6]. This results in a unique mechanical behavior whereby an imposed local displacement generates a diffused strain distribution throughout the structural network. Previous work has shown how a chiral honeycomb can be hosted within an airfoil profile to allow significant de-cambering deformations, which are obtained with the constitutive material remaining in the elastic range [7-9]. Such capabilities are numerically demonstrated in [9], and have been confirmed through a set of experiments performed on an airfoil prototype made of aluminum alloy [8]. A chiral wingbox with a polyamide chiral honeycomb core has been produced and benchmarked in [10] and dynamic properties of chiral truss-core assemblies have also been investigated [11]. The material selection for the analyses reported in $[8,9]$ was not expected to provide optimal performance, but rather, it was driven by manufacturing constraints. The studies in $[8,9]$ however show the potential benefits offered by chiral-core airfoils, and motivate the need for an effective manufacturing process that allows the use of composite materials. The design flexibility and the higher elastic limits of most composite materials have in fact the potential to significantly enhance the de-cambering capabilities of the airfoil, while providing practical relevance to the concept.

Numerical investigations performed as part of this study assess the response of an airfoil hosting a composite chiral structure. In addition, novel manufacturing techniques are developed to experimentally validate both mechanics and kinematics of the considered cellular configuration. The produced components are subjected to 
tensile and compressive loading to evaluate the onset of permanent deformations as well as displacements and loads leading to failure, thus highlighting critical locations within the manufactured chiral topology. Finally, detailed explicit Finite Element (FE) analyses are employed to predict the response and failure mechanisms of the manufactured assemblies.

The paper is organized in six sections including this introduction. Section 2 reports the preliminary numerical evaluations to investigate the morphing properties of a composite chiral airfoil. Sections 3 and 4 describe the manufacturing process and the mechanical tests of composite chiral structural components, respectively. Section 5 presents the numerical approach to model the response and failure of this type of structures by using detailed non-linear finite element models of the manufactured components. Concluding remarks are finally presented in Section 6 .

\section{Morphing properties of a composite chiral airfoil}

This section presents numerical investigations on the morphing properties of a bi-dimensional airfoil, representing a possible embodiment of the chiral structural concept. On the basis of the results presented in [8,9], a FE model of the airfoil hosting a chiral core is developed. The application that is considered in the model exploits the deformability of the core to change the camber of the airfoil [7-9]. A reduction of the camber (de-cambering effect) and a consequent modification of the airfoil performances is obtained if the aerodynamic load on the airfoil is increased. The computations that are presented are based on finite deformations and consider a concentrated load at the trailing edge of the airfoil, to reproduce the experimental setup discussed in [8]. Displacement levels corresponding to the onset of inelastic phenomena at one location within the structure are considered as measures of the morphing capabilities of the modeled configurations. De-cambering deflections obtained with a chiral core made of carbon/epoxy laminates are compared with those achieved with a metallic structure.

\subsection{The chiral geometry}

The chiral networks are obtained through the assembly of circular elements (nodes), connected by ribs (ligaments) tangent to the nodes. The parameters defining the chiral geometry are $R, L, \beta, t_{b}$, and $t_{c}$, which in turn denote the distance between the node centers, the rib length, the angle between the imaginary line connecting the circles and a rib and the node and ligament wall thickness (Fig. 1).

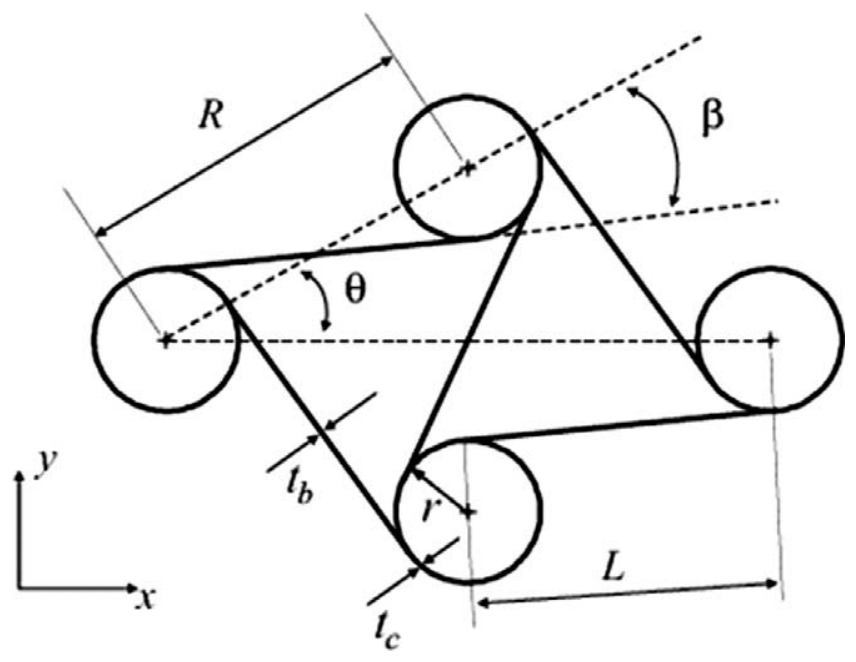

Fig. 1. Chiral topology.
These parameters are related as follows: $\tan \beta=2 r / L, \sin \beta=2 r / R$, and $\sin \beta=1 / 2$ [6]. The chiral geometry is significantly altered by changes in $L / R(L / R=\cos \beta)$ here denoted as the "topology parameter", which in turn strongly affects the mechanical behavior of the proposed truss-core airfoils $[8,9]$.

\subsection{Airfoil configurations}

An Eppler 420 profile hosts a truss-type structure according to the configuration shown in Fig. 2. The choice of the airfoil, which was made in previous investigations [8,9], is driven by its aerodynamic shape with ensures theoretically high-lift at low free-stream velocity and a tendency to achieve a structurally-stable low camber, low-induced drag configuration as the free-stream velocity increases.

The core layout is obtained through a linear mapping of an initially periodic chiral lattice into the airfoil as described in $[8,9]$. In its periodic configuration, i.e. before its mapping into the airfoil, the lattice features a nominal topology parameter $L / R \sim 0.60$. For simplicity, the leading and the trailing edges are considered made of light alloy $\mathrm{Al} \mathrm{6061-T051} \mathrm{with} \mathrm{an} \mathrm{out-of-plane} \mathrm{thickness} \mathrm{of}$ $19 \mathrm{~mm}$ (Fig. 2), and are correspondingly very stiff in comparison to the core. The airfoil has a chord $c=0.7 \mathrm{~m}$, which replicates the dimensions previously used for the experimental analysis presented in [9] and whose choice was dictated by manufacturing constraints.

Two materials are used for the analysis: namely an aluminum alloy T6061-T051 and laminated carbon/epoxy fabric plies. The properties for each of the investigated materials are reported in Table 1 . The core density of the proposed assemblies certainly affects their de-cambering compliance, which is found to be inversely proportional to the core relative density $[8,9]$. As a result, three distinct configurations characterized by 2,3 , and 4 cells across the airfoil thickness are analyzed (Fig. 3). All considered configurations feature a node wall thickness $t_{c}=1.4 \mathrm{~mm}$ and a ligament wall thickness $t_{b}=0.8 \mathrm{~mm}$.

\subsection{Finite element model}

Finite element models of the considered airfoils are developed using the commercially available software ANSYS ${ }^{\circledR}$, and include both beam and plane elements to discretize the core and the leading edge respectively. For simplicity, the airfoil is considered completely clamped at the leading edge, which therefore is not modeled for the de-cambering analysis presented below. Specifi-

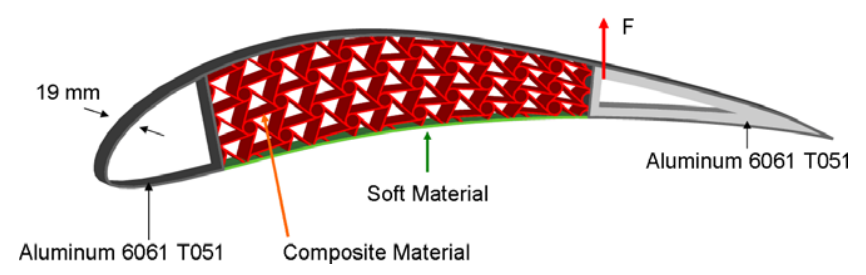

Fig. 2. Chiral core airfoil.

Table 1

Elastic constants of considered materials.

\begin{tabular}{lll}
\hline & $\begin{array}{l}\text { Carbon-fiber } \\
\text { CC90/ET443 SEAL }\end{array}$ & $\begin{array}{l}\text { Aluminum } \\
\text { Al 6061-T051 }\end{array}$ \\
\hline$E_{x x}$ & $56.6 \mathrm{GPa}$ & $68.9 \mathrm{GPa}$ \\
$E_{y y}$ & $56.6 \mathrm{GPa}$ & $68.9 \mathrm{GPa}$ \\
$v_{y x}$ & 0.0514 & 0.333 \\
$G_{x y}$ & $4.043 \mathrm{GPa}$ & $27.6 \mathrm{GPa}$ \\
$\varepsilon_{11 \max }$ & 0.009 & 0.004 \\
\hline
\end{tabular}



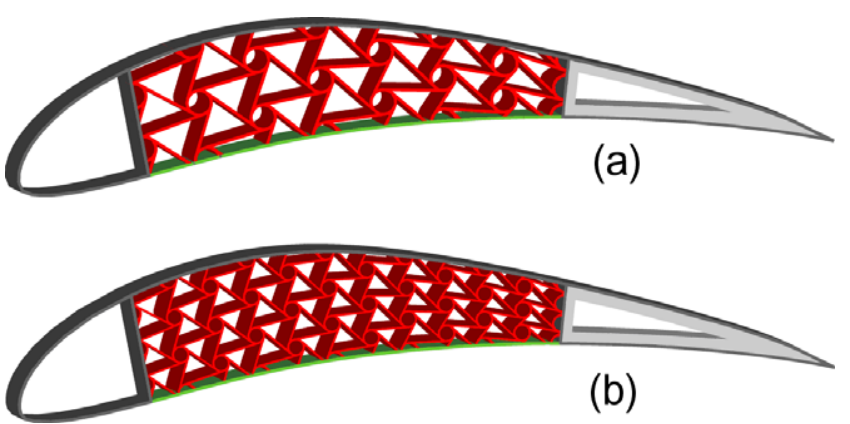

Fig. 3. Examples of considered configurations with 2 cells (a) and 3 cells (b) across the airfoil thickness.

cally, Timoshenko beam elements with finite-strain capabilities are used for the core and outer-profile, while plane elements are assigned to the solid-like trailing-edge section $[8,12]$. In order to allow de-cambering deformations, the upper and lower portions of the airfoil profile are modeled as a soft material with stiffness 100 times lower than that of the core.

The developed model predicts the de-cambering characteristics of the airfoil corresponding to an imposed concentrated load at the trailing edge as shown in Fig. 2. While such conditions do not reproduce a typical aerodynamic load, they are consistent with the experiments carried out in $[8,9]$, for which the same numerical models employed here showed excellent predictive capabilities. The large de-cambering deformations are estimated by considering a linear material, through a non-linear analysis which includes geometric stiffening and employs finite-strain elements. Equilibrium is computed via the implicit Newton-Raphson method. Such procedure allows the monitoring of the evolution of strain in the chiral network as well as the identification of the displacement levels corresponding to the limits of the elastic behavior for the considered materials. The constituent materials are considered linearly elastic, with the onset of the plastic deformation being identified by the onset of the yield stress for the aluminum alloy. A maximum strain criterion is instead chosen for the composites, with the maximum strain set to $\varepsilon_{11 \max }=0.009$ in the fiber-reinforcement direction of the carbon/epoxy plies, for both tension and compression. Such limit is lower than the tensile strain at failure in the fiber direction reported for most of the carbon/epoxy materials adopted in advanced structural design [13]. It can be considered as adequate for the objective of the computations and is consistent with the choice to adopt the yield strain for the light alloy without introducing any safety margin.

\subsection{Numerical results}

The influence of core material as well as core density on airfoil de-cambering compliance is depicted in Fig. 4, which presents the trailing-edge displacement resulting from the applied concentrated load up to the onset of limit strain conditions anywhere in the assemblies. The low core density associated with 2-cell configurations yields the highest compliance while 4-cell configurations yield the highest stiffness, for all considered materials. These results are consistent with the findings in $[8,9]$ which illustrate how larger nodes tend to induce larger bending of the ligaments, which is the leading deformation mechanism for the considered assembly.

The maximum loads of the curves reported in Fig. 4 correspond to the onset of inelastic mechanisms anywhere in the structure. Hence, the curves allow identifying the maximum trailing-edge displacements that can be reached without exceeding the imposed strain limits in the modeled structures. Such displacement is indicated for each curve reported in Fig. 4 in terms of chord percentage. Figs. 5 and 6 show the axial stress distribution at the limit of the elastic regime of the material for the light alloy airfoils and the composite airfoils, respectively. The results are presented as contours along the axes of the beams, which have been thickened in the figures to better visualize the stress maps. Overall, the results describe the morphing capabilities of the chiral structural concept. The use of carbon composite as core material allows a trailing-edge displacement which is generally twice as large as that obtained with aluminum alloy at the onset of plasticity. Such results is directly related to the higher allowable strains considered for the composite material and it could be observed that composites with even higher strain at failure, such as unidirectional carbon tapes or glass fiber reinforced composite, could be adopted.

While the dependency of trailing-edge displacement upon core density and material can be considered as intuitive, it can be observed that the associated distribution of stress at the limit of elastic range can be influenced by the choice of the constituent material. In particular, the highest stresses for the 2-cell aluminum configuration (Fig. 5a) appear confined at the lower portion of the core, while the same configuration made of carbon-fiber features highest axial stresses in the ligaments near the upper portion of the profile and in those closest to the leading edge. Actually the two stress distributions are not referred to the same geometrical configuration. Infact, computation fully takes into account geometrical non-linearities and the displacement at the elastic limit for the composite core is much higher than the one for the light alloy airfoil, as reported in Fig. 4. Finally, it should be noted that, as in [8], the core nodes exhibit much lower stress than the ligaments.
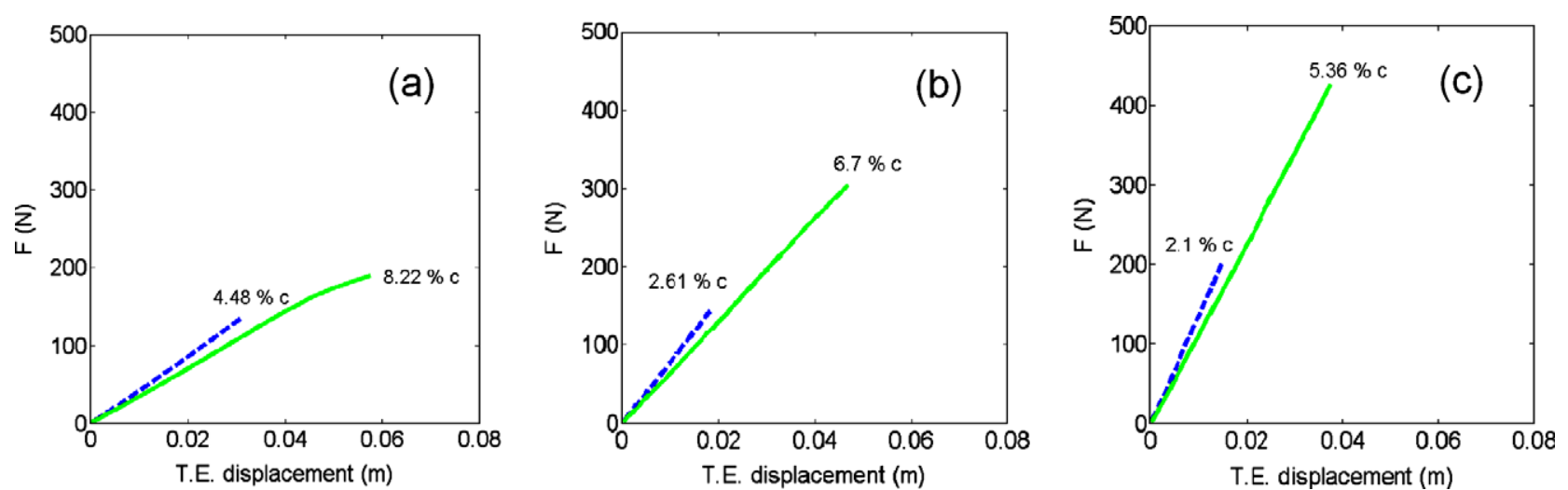

Fig. 4. Trailing-edge displacement vs. applied load for: (a) 2-cell configuration, (b) 3 cells, and (c) 4 cells. Aluminum alloy blue line and carbon fiber green line. 
(a)

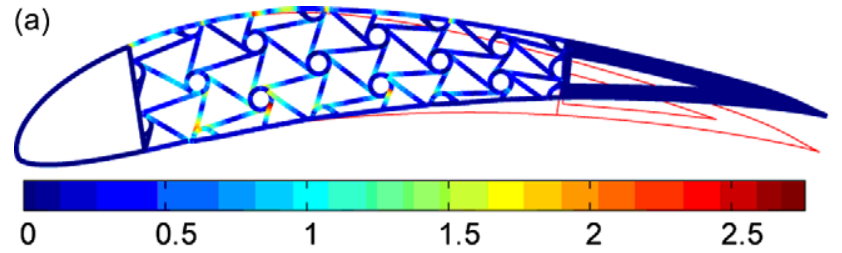

(b)
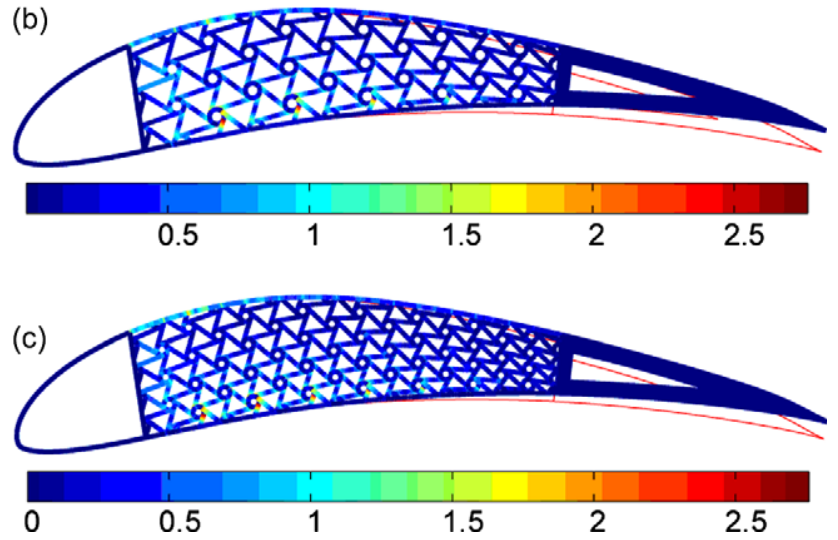

Fig. 5. Axial stress distribution $(\times 100 \mathrm{MPa})$ at the limit of the elastic regime of the material for aluminum alloy airfoil: (a) 2-cell configuration, (b) 3 cells, and (c) 4 cells.
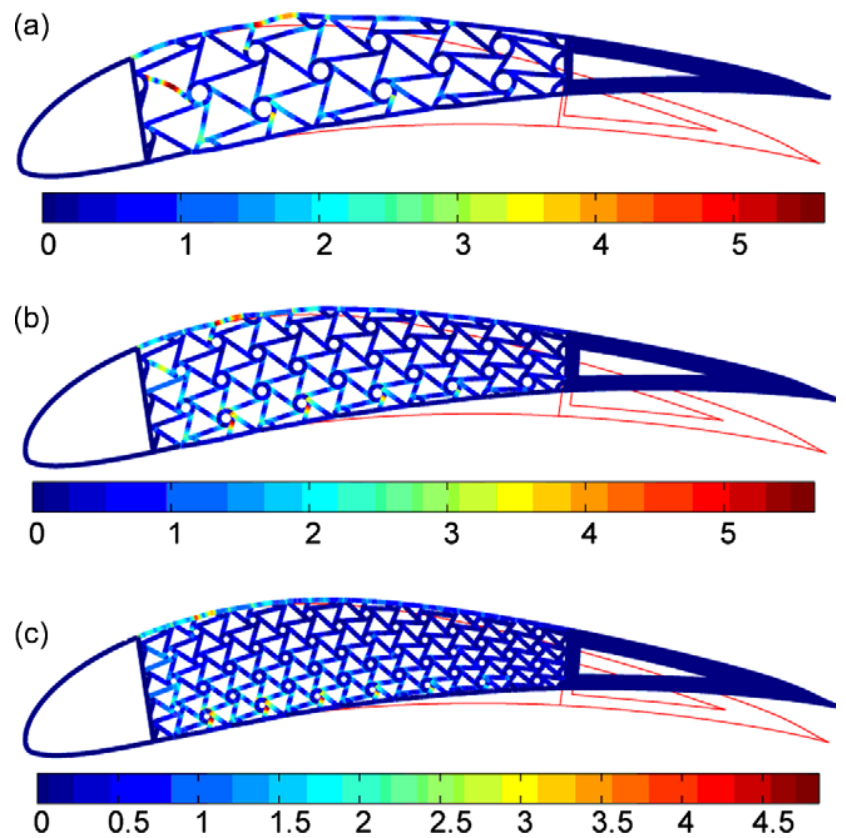

Fig. 6. Axial stress distribution $(\times 100 \mathrm{MPa})$ at the limit of the elastic regime of the material for carbon fiber airfoil: (a) 2-cell configuration, (b) 3 cells, and (c) 4 cells.

\section{Manufacturing process}

The previous results confirm the potential morphing capability of an airfoil structure hosting a composite chiral core and motivate the development of a technological process to produce such types of unconventional structures. The set up of a feasible manufacturing process is focused on the production of the chiral component shown in Fig. 7. Such element cannot fully represent the response of a chiral cell in a multi-cell structure, because the six external nodes, which are linked only to three ligaments, will not behave as in a regular chiral topology. However, the considered structural

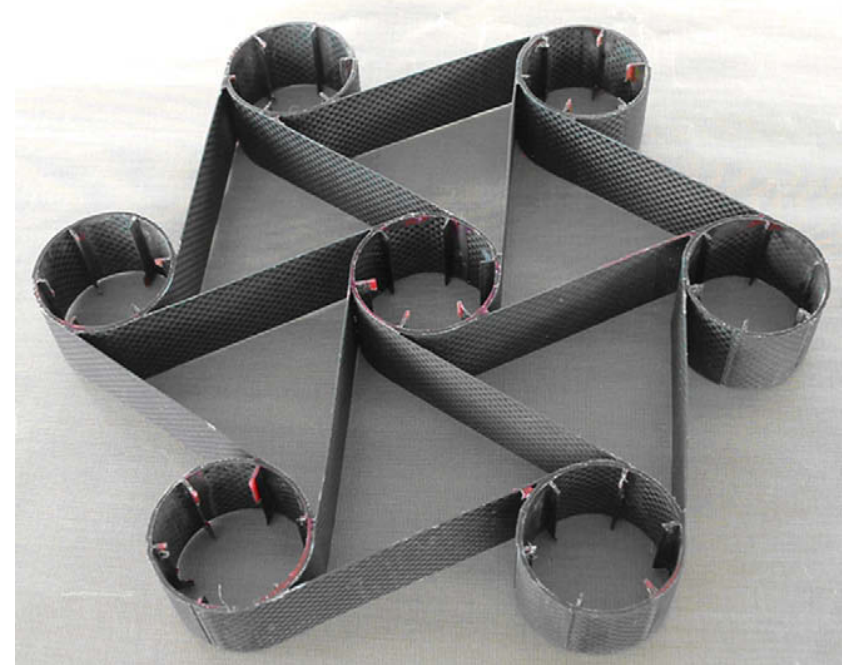

Fig. 7. Chiral composite element.

component will represent a useful benchmark to set up a possible manufacturing process for composite chiral structures and to experimentally investigate some basic aspects of their mechanical response. The element is characterized by the following parameters $r=18.0 \mathrm{~mm}, R=83.3 \mathrm{~mm}, L=75 \mathrm{~mm}$, by an overall in-plane dimension of $170 \mathrm{~mm}$ and an out-of-plane depth of $25 \mathrm{~mm}$. Such relatively large dimensions have been chosen to avoid excessive complications due to material handling and ply drapability during the set up phase of the process.

The developed process consists of a first step for the production of thin curved laminates according to a classical vacuum bag technology and of a subsequent phase to assemble and bond the laminates to obtain the chiral topology.

Ligaments have been manufactured using CC90/ET443 SEAL plain weave carbon fabric plies, with a cure ply thickness of $0.100 \mathrm{~mm}$. A $[0]_{5}$ lay-up sequence has been selected. The material properties have been already presented in Table 1 as far as stiffness parameters are concerned, while the reported maximum allowable strain should be considered a reasonably conservative estimate for the strength along the fiber-reinforcement direction. Fig. 8 shows the mould used in the first step of the process and the curved laminate cured for the production of the $25 \mathrm{~mm}$ wide ligaments subsequently cut from such laminate.

The previously described numerical analyses of the airfoils confirmed that nodes are only slightly deformed and tend to rigidly

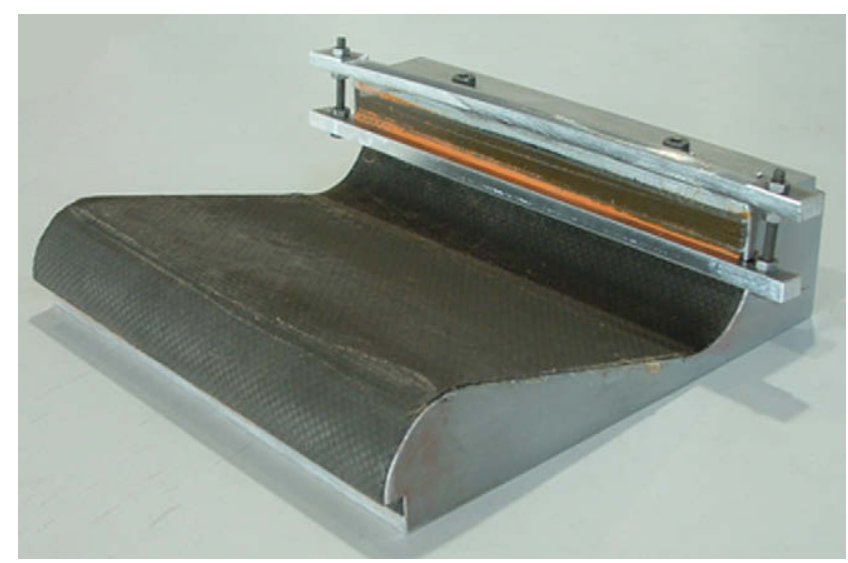

Fig. 8. Mould for the production of ligaments. 


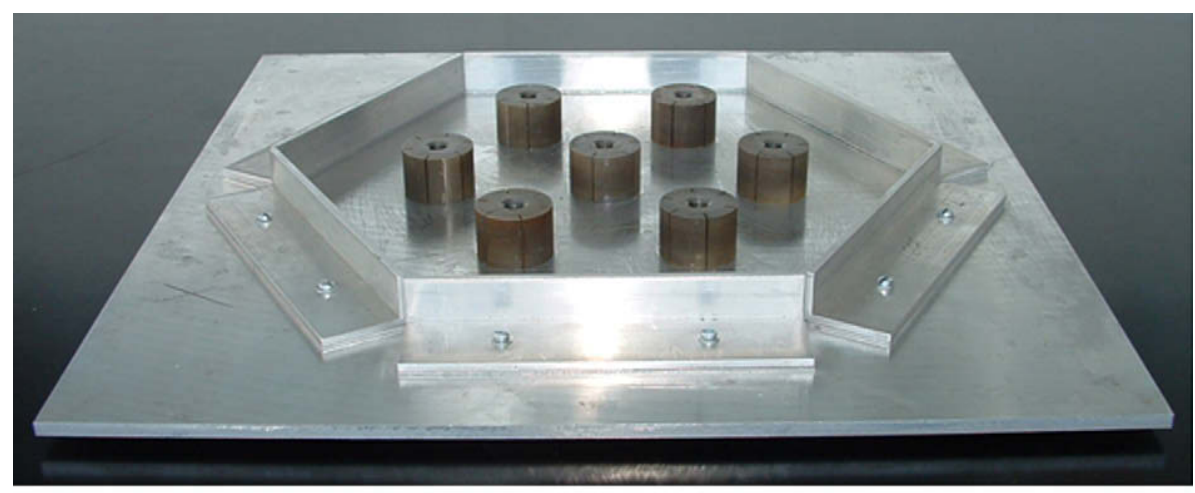

(a)

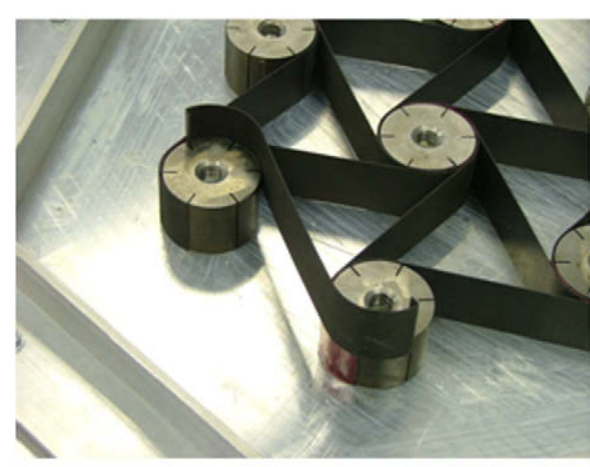

(b)

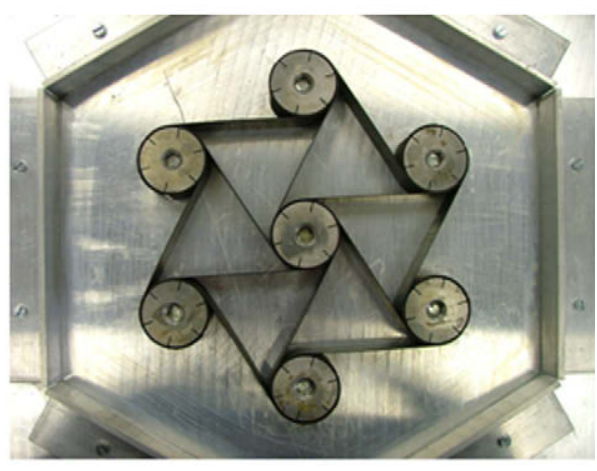

(c)

Fig. 9. Mould for component assembly (a), positioning of ligaments (b) and assembled component (c).

(a)

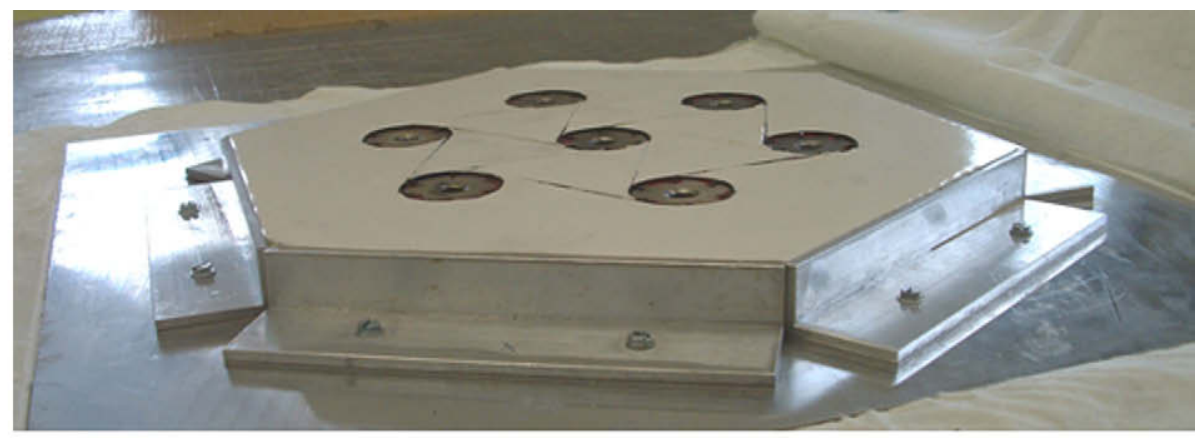

(b)

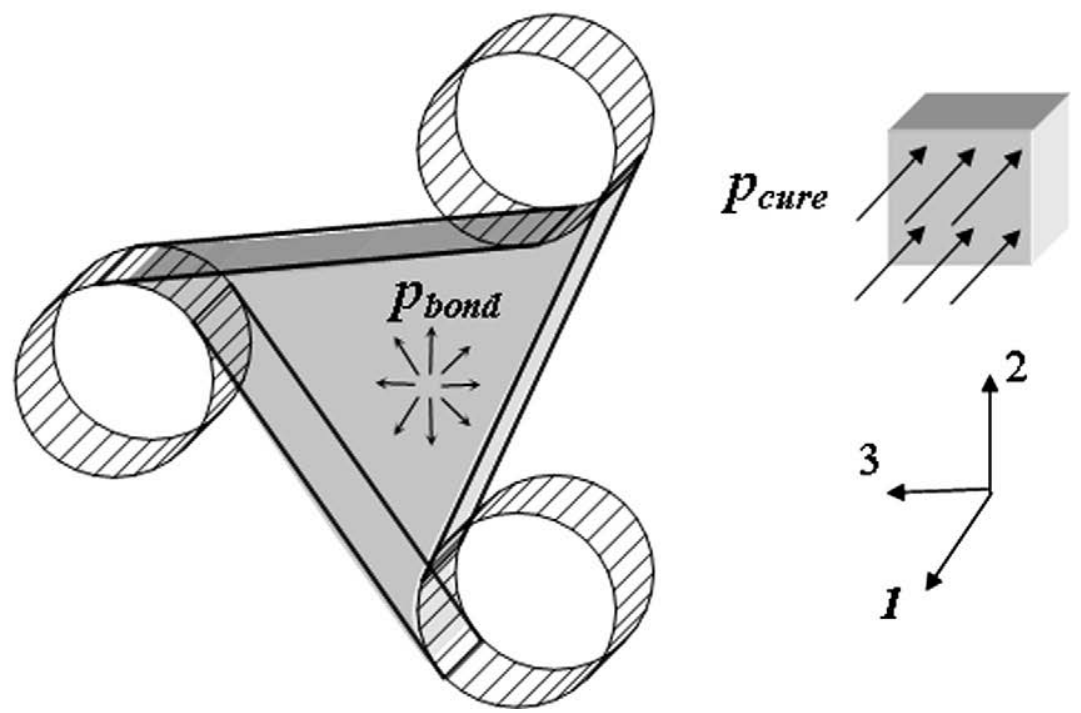

Fig. 10. Production of silicon rubber blocks (a) and application of the pressure during bonding (b). 
rotate so that they can be considered not particularly critical from the structural point of view. Such considerations lead to investigate a manufacturing solution simply consisting of an assembly of ligaments, without separately producing the nodes that are obtained by superimposing the curved ends of the ligaments.

Accordingly, the second step of the process includes the assembling of the chiral element and a second curing cycle to bond the ligaments by means of a structural adhesive film. An epoxy adhesive AFK-163-2K has been employed. For this step an hexagonal mould featuring a series of metallic cylinders has been designed (Fig. 9a). The curved end of the ligaments is wrapped around the metallic cylinders of the mould (see Fig. 9b), which are equipped with a series of slot in which flanges on the ligaments are inserted for accurate positioning.

The strength of the obtained chiral structure strongly depends on the quality of the adhesion between its parts. The application of a proper pressure between the ligaments, assembled in the composite chiral component as shown in Fig. 9c, is a critical aspect of the manufacturing process. This has been addressed by carrying out the adhesive curing in autoclave, by applying a vacuum bag technique, and by exploiting the elastomeric tooling technique [14] by means of silicon rubber blocks which have been introduced between the ligaments. Such blocks have been casted by pouring and allowing curing by condensation of a liquid silicon rubber compound into the mould, as presented in Fig. 10a. The mould is prepared for the final curing process by cutting strips of structural adhesive film and by laying them on the curved ends of the ligaments. The ligaments are then assembled on the metallic cylinders and the solid silicon rubber blocks are positioned in the inter-ligament voids. The assembly is subsequently introduced in a vacuum bag and prepared for the curing cycle in autoclave.

The pressure application mechanism is shown in Fig. 10b. The high castability of the adopted silicon rubber, a RECKLI ${ }^{\mathrm{TM}}$ SI compound 20 having a Shore A hardness of 20 , allowed an accurate reproduction of the shape of the voids between the ligaments in the assembly. The idealization presented in Fig. 10b assumes a pressure $p_{\text {cure }}$ acting on the filling material constrained in the 2, 3 plane by the ligaments that are considered rigid. By assuming isotropic thermo-elastic material behavior and by considering $\sigma_{11}=-p_{\text {cure }}$ and $\varepsilon_{22}=\varepsilon_{33}=0$, the pressure $p_{\text {bond }}$ exerted in 2,3 directions can be approximately evaluated as:

$p_{\text {bond }}=-\sigma_{33}=-\sigma_{22}=\frac{E_{r}}{1-v_{r}} \alpha_{r} \Delta T+\frac{v_{r}}{1-v_{r}} p_{\text {cure }}$

where $E_{r}, v_{r}$ are the elastic parameters of the rubber, $\alpha_{r}$ its coefficient of thermal expansion (CTE) and $\Delta T$ the difference between curing and ambient temperature. Tensile tests and ThermoMechanical Analysis (TMA) performed on the silicon rubber provide estimates for the elastic modulus $E_{r}=0.449 \mathrm{MPa}$ and a coefficient of thermal expansion $\alpha_{r}=4.72 \mathrm{e}-041 / \mathrm{K}$. The values of $E_{r}$ and $\alpha_{r}$, which are functions of the rubber chemical composition, are consistent with the data reported in $[14,15]$ for soft silicon rubbers. The high Poisson coefficient of silicon rubber allows maximizing the pressure exerted on the ligaments and the considerably high CTE coefficient gives a further contribution. Assuming a Poisson coefficient $v_{r}=0.49$ and a pressure $p_{\text {cure }}=3 \mathrm{bar}$, the pressure $p_{\text {bond }}$ turns out to be 3.24 bar, with a contribution of 0.35 bar due to the thermal expansion of the rubber.

The developed technique allows the production of composite chiral elements of good manufacturing quality, as estimated through simple visual inspections, also considering that the nodes of the components previously manufactured without the insertion of silicon blocks exhibited voids and even complete debonding between the superimposed ligaments. Although this initial attempt has considered a chiral geometry of relatively large dimensions, it is fully expected that this approach lends itself to the manufacturing of chiral topologies with smaller cells. Nevertheless, the drapability of the pre-preg material as well as the need to assembly very small composite parts are issues that would need to be carefully addressed and which may complicate the application of the manufacturing process to smaller cell sizes.

\section{Mechanical tests of manufactured components}

This section describes the set of mechanical tests performed to estimate the mechanical behavior of the manufacturing process. Three components have been produced by applying the previously described process. The strength and the compliance of such chiral composite elements have been assessed by means of tests consisting of tensile and compressive cycles performed at very low velocity. The experimental activities had the objective of measuring the capability of the manufactured components to undergo large displacement without experiencing permanent deformations, degradation or failures. In addition, non-linear geometrical effects in the response of chiral composite structures, the possible failure modes and weak points of the structural assembly are also investigated. Relevant data for the set up and the validation of numerical approaches have also been collected.

\subsection{Test set-up}

To accomplish such objectives, a simple test lay-out has been considered, whereby two opposite cylinders of the composite chiral components have been respectively connected to the moveable and the fixed cross head of an MTS 858 Test System (Fig. 11). The connecting pins have been inserted in rings having the outer diameter matching the inner diameter of the cylinders and are allowed to freely rotate about their axis. Each one of the three components has been subjected to a different loading-unloading cycle, according to the displacement time histories reported in Fig. 12. A cross head speed of $2 \mathrm{~mm} / \mathrm{min}$ has been considered for all the tests. The imposed displacement histories allowed the detection of possible stiffness degradations or permanent deformations occurring after the first loading-unloading cycle. Tests have been continued until failure of the component, with tensile or compressive failures

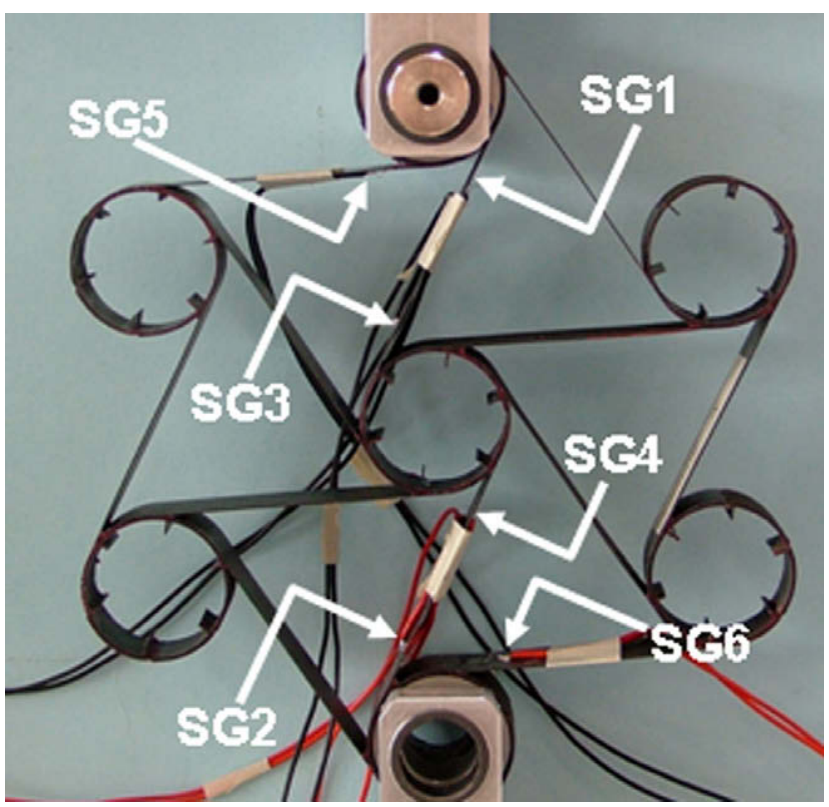

Fig. 11. Test set-up. 


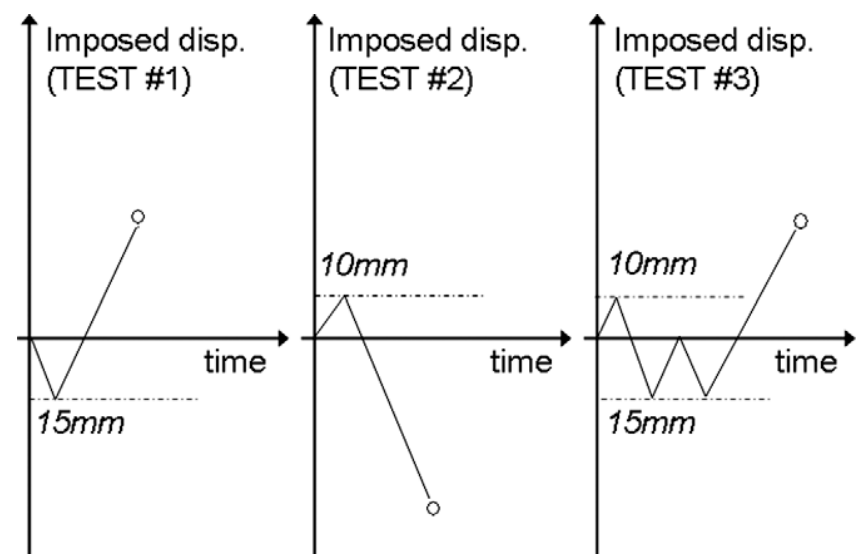

Fig. 12. Loading-unloading cycles performed during the tests.

that allowed to quantify the component strength and to identify the actual weak points of the assembled structure. The components produced in the first manufacturing attempts were deformed and it was estimated that the two ligaments aligned closest to the vertical direction and the almost horizontal ligaments close to the constrained nodes would be the most critically deflected during the tests. Hence, in the tests on the first two components, six strain

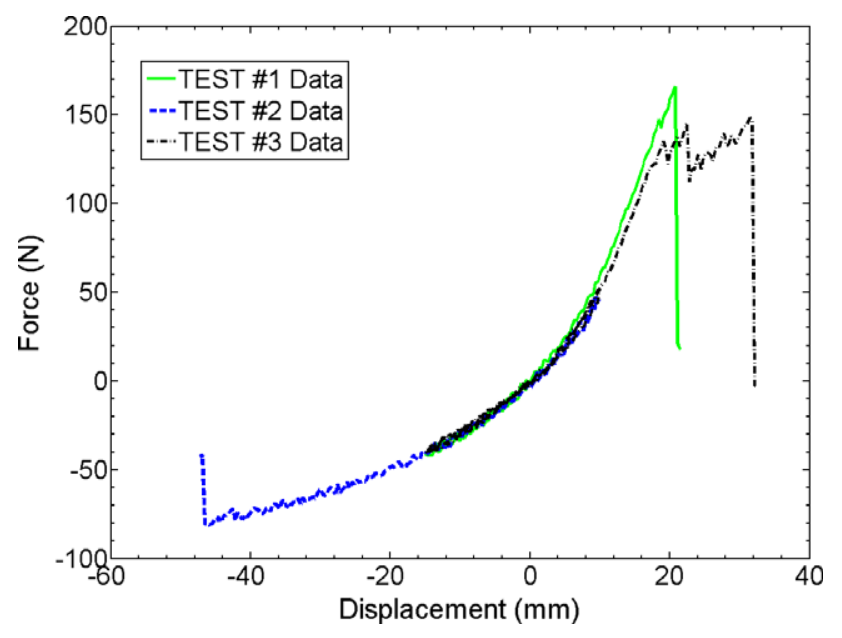

Fig. 13. Experimental force vs. displacement responses of the chiral composite element.

gauges have been installed according to the scheme reported in Fig. 11. Although more gauges have been installed on the third component, the most interesting data were generally acquired by the gauges in the positions shown in Fig. 11.

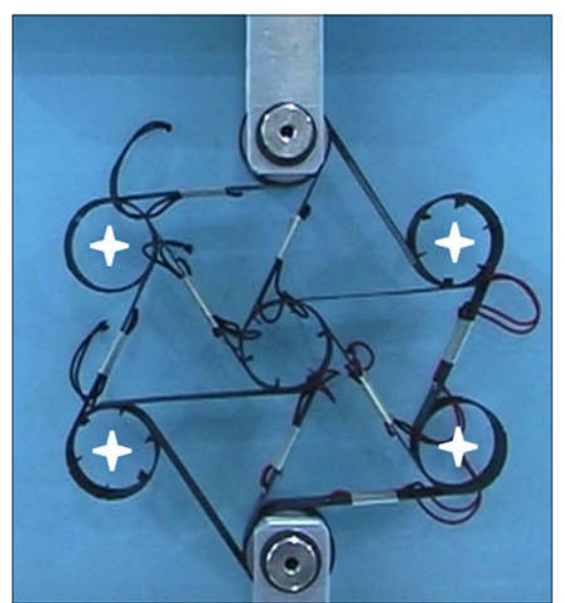

(a)

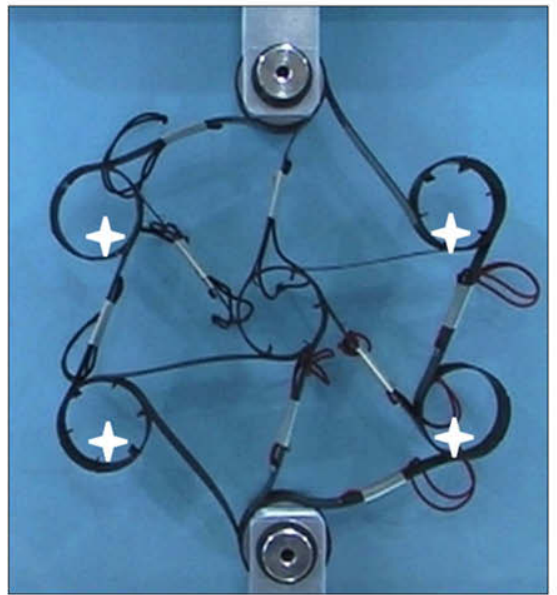

(c)

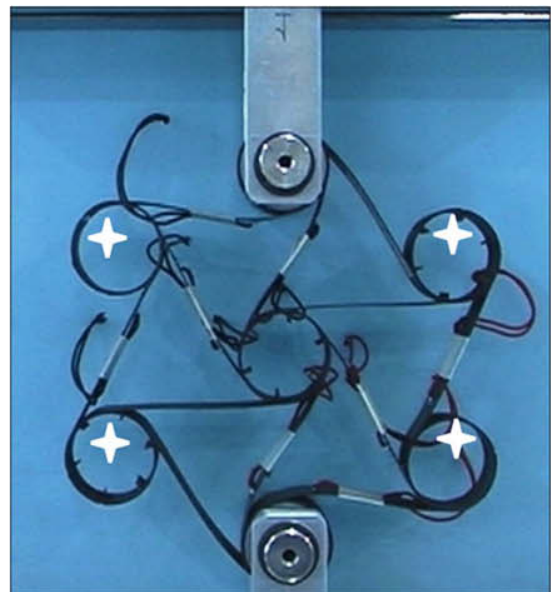

(b)

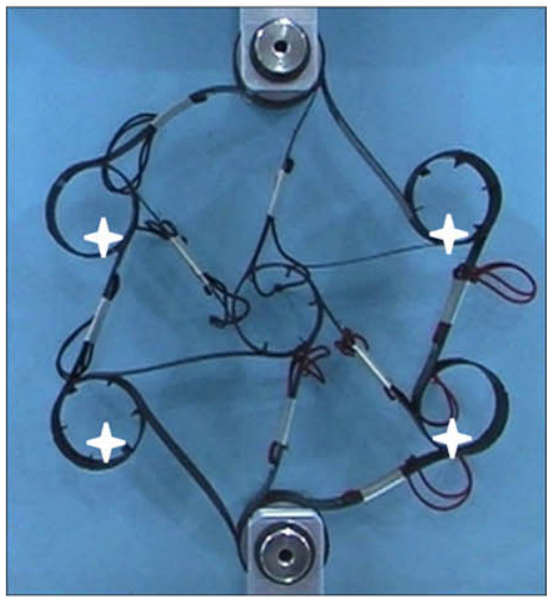

(d)

Fig. 14. TEST \#1: initial geometry (a), deformation at maximum compressive displacement (b), deformation during the tensile phase (c), deformation at failure (d). 


\subsection{Result and discussions}

Fig. 13 reports the force vs. displacement responses measured during the three performed tests. All the curves show a non-linear behavior and a tendency to soften in compression and to stiffen in tension. Due to the imposed displacement histories, several loading-unloading responses are superimposed in the range $-15 \mathrm{~mm}$ $\div 10 \mathrm{~mm}$. As far as tensile response is concerned, the behavior recorded in TEST \# 1 and TEST \#3 has been obtained after having carried out respectively one and two loading-unloading compressive cycles (see Fig. 12). In such preliminary compressive cycles the imposed compressive displacement of $15 \mathrm{~mm}$ corresponds to about $9 \%$ of the cell dimension. The comparison of the tensile response obtained in TEST \#1 and TEST \#3, after the compressive cycles, with the tensile cycle of TEST \#2, performed on the cell before any other loading-unloading cycle, shows that the compressive cycles have not induced significant degradation of the component stiffness. Similarly, the tensile loading-unloading cycle, performed on the item used in TEST \#2, has not induced significant differences in the subsequent compressive response with respect to TEST \#1 and TEST \#3. The preliminary tensile cycle carried out before the compressive loading in TEST \#2 has been performed imposing a tensile displacements of $10 \mathrm{~mm}$, which corresponds to about $6 \%$ of the component dimensions. Although identical force vs. displacement paths have been followed in the loading and unloading phases of the quasi-static cycles that have been performed, the occurrence of hysteretic effects cannot be excluded at high load application rates.

Fig. 14 presents a sequence taken from TEST \#3. The compliance of the structure is well shown by the figures that have been taken by a fixed camera. The position of the centers of each node in the undeformed configuration has been marked by white symbols which have been superimposed to the deformed configurations in Figs. 14b-d. A qualitative tendency to transversely contract during the compression and expand during the tension can be observed, which is in accordance with the theoretical negative Poisson ratio expected for chiral honeycombs $[5,6]$.

In TEST \#1 final failure occurred in tension for debonding of the ligaments at the upper cylinder in correspondence of a displacement of $20 \mathrm{~mm}$, about $12 \%$ of the cell dimension. Fig. 15a shows the detail of the failure that occurred with a sudden drop of load carrying capacity, although an initial deviation from the previous regular course can be detected in Fig. 13 at a displacement of $18 \mathrm{~mm}$. The tensile failure obtained in TEST \#3 is reported in Fig. 15b. The force vs. displacement curve referred to TEST \#3 shows a deviation from the regular course at about $18 \mathrm{~mm}$, as in TEST \#1 (see Fig. 13). As in the previously described test, such deviation corresponds to the onset of a debonding process of the ligaments close to the upper and lower constrained cylinders. However, a different post-failure response has been recorded in TEST \#3, characterized by a phase of progressive debonding of the superimposed part of the ligaments. The corresponding force vs. displacement response shows that the load carrying capacity of the composite component remained close to the maximum load level until a displacement of $30 \mathrm{~mm}$, before a final load drop (Fig. 13). Despite such differences in the post-failure response, it can be observed that the maximum load levels carried by the items in TEST \#1 and TEST \#3 are similar.

Fig. 16 shows the variation of the strain as recorded by the gauges in TEST \#1. Only the signals acquired by the gauges SG2, SG4 and SG6, located in the lower part of the component (see Fig. 11), are reported. The variation of the SG1, SG3 and SG5 signals, acquired at the same location in the upper part of the cell show very similar trends are here omitted for sake of brevity. During the tensile phase of the test, the highest levels of strain have been recorded by the gauges SG2 and SG6, and identically by SG1 and SG5. Such gauges have been located on the compressed side of the ligaments close to the constrained cylinders. Fig. 14c and $\mathrm{d}$ confirm that such ligaments can experience severe bending at the strain gauges locations during the tensile phase of the tests. It is worth noting that the maximum absolute values of the obtained strain levels, reported in Fig. 16, matches the maximum admissible strain considered in Table 1 for the carbon fabric

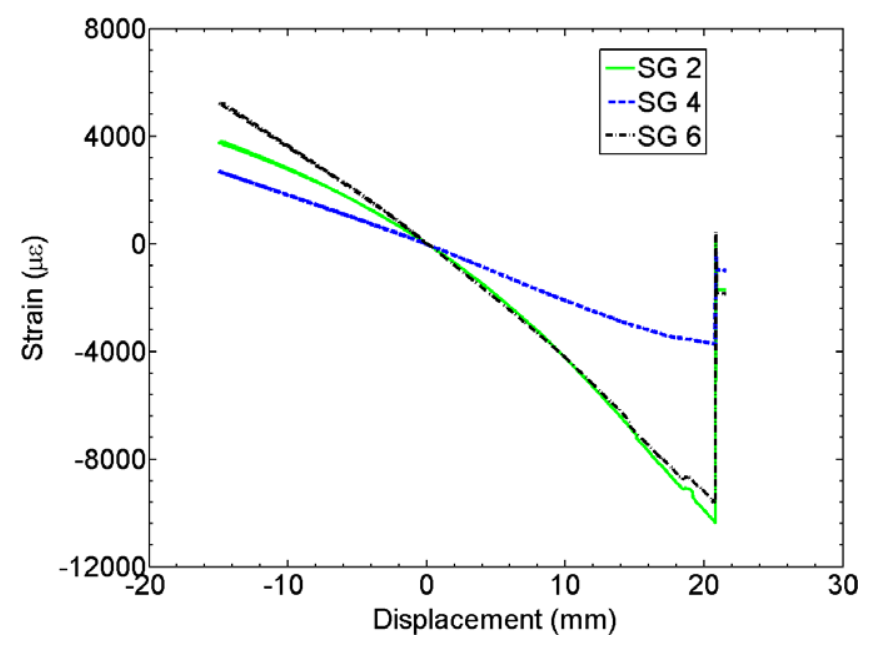

Fig. 16. TEST \#1: strain vs. displacement response.

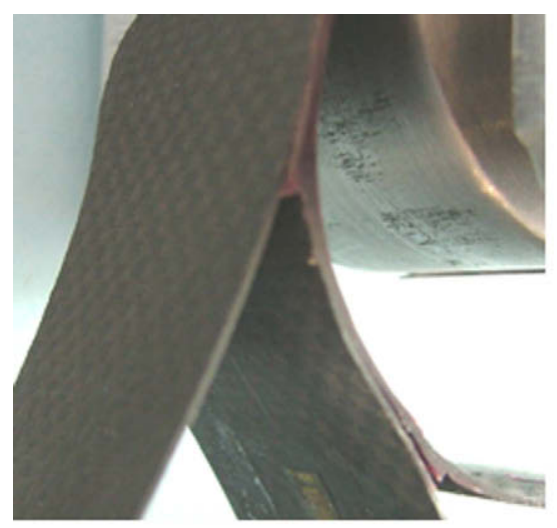

(a)

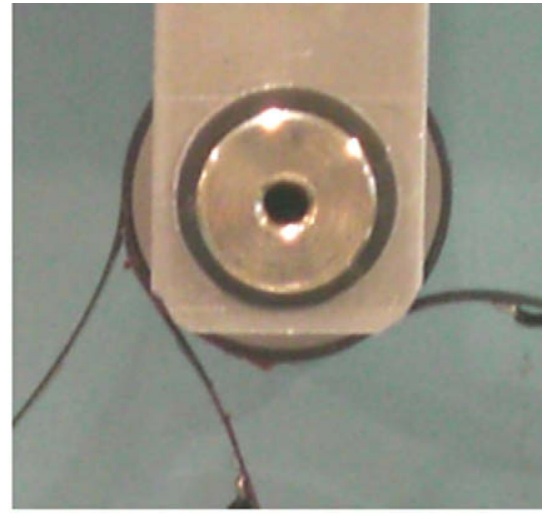

(b)

Fig. 15. Tensile failures: TEST \#1 (a) and TEST \#3 (b). 
material. Hence, although the presented results indicate that bonding is the actual weak point of the manufactured structures in tension, the bonding strength appears adequate to exploit almost the whole in-plane compressive strength of the composite ligaments.

As far the compressive failure is concerned, the force vs. displacement curve referred to TEST \#2 in Fig. 13 indicates that failure has been obtained at a displacement of almost $-50 \mathrm{~mm}$, corresponding to about $30 \%$ of the cell dimension. Fig. 17 reports a sequence taken from TEST \#2 showing the overall level of contraction of the item obtained in the experiment. Failure has been obtained, in this case, for excessive bending of the ligaments in the two points evidenced in Fig. 17b. Fig. 17c shows one of the constrained cylinder of the composite element, after the removal from the test fixture. The failure of the ligament and the absence of visible damage in the bonding between the ligaments are apparent. Fig. 18 shows strain signals recorded in TEST \#2 by SG2, SG4, SG5 and SG6. It can be observed that the gauges SG5 and SG6, located on the tensile side of the failed ligaments have actually experienced the highest strain levels among the recorded ones. The failure of the ligaments have occurred in correspondence of strain levels between 0.013 and 0.014 , thus confirming that the limit of 0.009 set in Table 1 can be considered a reasonable and conservative evaluation for the material strength. Overall, compression appears less critical than tension as far as the cell strength is concerned. The visual inspection of the cell have not provided

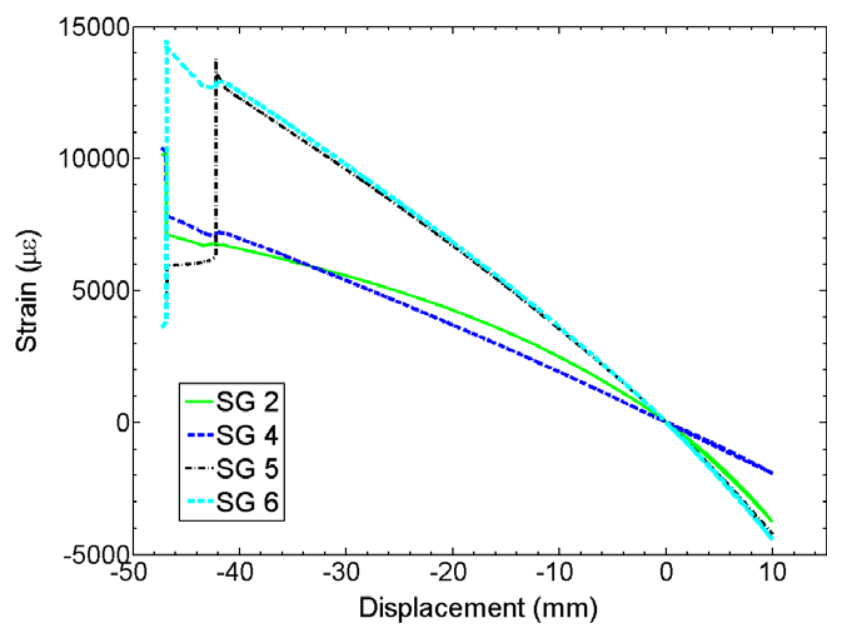

Fig. 18. TEST \#2: strain vs. displacement response.

evidence of any damage in the adhesive film, so that the weak point of the structure can be reasonably considered the bending strength of composite ligaments.

It may be concluded that the performed experiments point out the highly non-linear response and two main possible failure

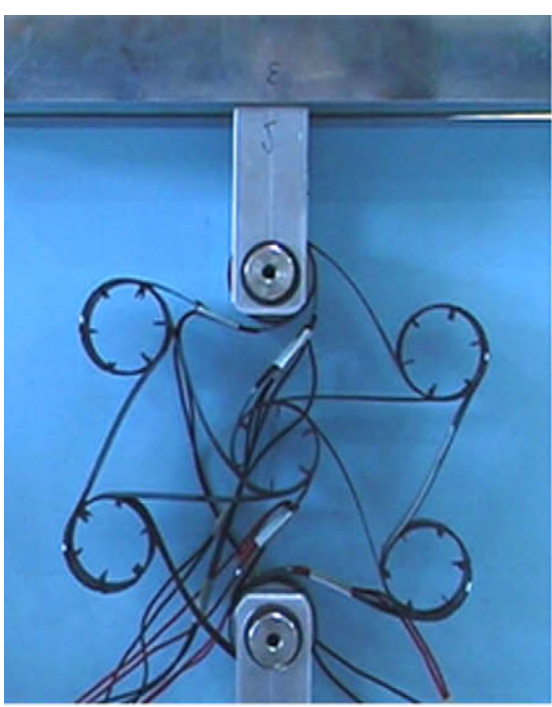

(a)

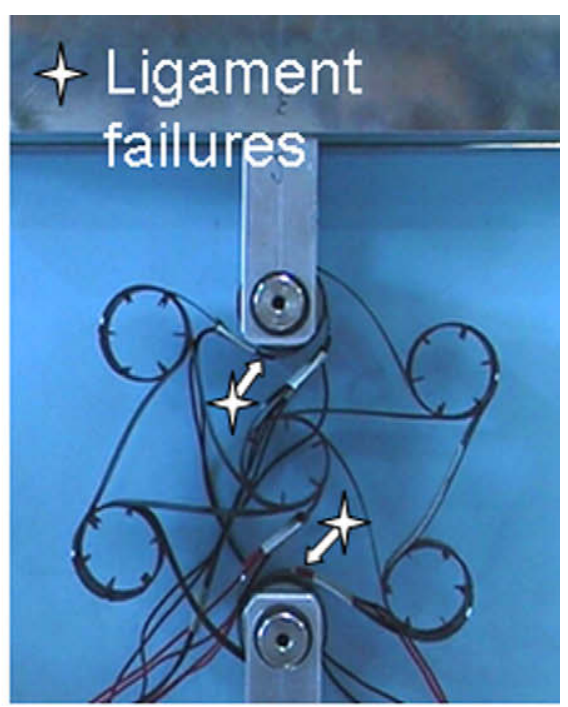

(b)

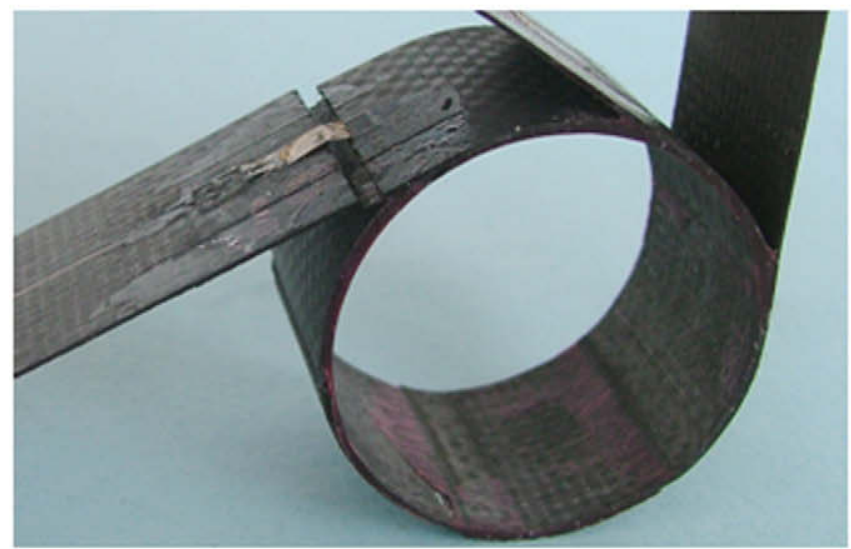

(c)

Fig. 17. TEST \#2: deformation at $35 \mathrm{~mm}$ of displacement (a), at failure (b) and compressive failure mode (c). 
modes of the composite chiral structures manufactured according to the developed process: bending failure of the ligaments, typically originated by a compressive failure of the plies, and debonding between ligaments. Both failure modes are triggered by ligament bending. If bending act to wind the ligament onto the node, ligament failure occurs, but when bending act in the opposite direction, debonding can be triggered.

\section{Numerical approach for strength predictions}

The possibility to numerically evaluate the non-linear response as well as the strength levels of the presented chiral structures can play a key role in the development of structures hosting composite chiral cores. Basing on the results obtained in the experiments, the bending levels experienced by the ligaments can be adopted as failure indices of the composite structure. Such bending levels could be evaluated by models developed at the structural scale, like the ones presented in Section 2 of this work, under given loading conditions. The possibility to numerically evaluate the strength of simple components by means of a detailed non-linear model would significantly reduce the experimental activity required for each design hypothesis. This section describes the development of a numerical approach aimed at such evaluation. The approach is first presented considering the basic and theoretical aspects. The tests performed to characterize the adhesive toughness are subsequently presented. The numerical approach is first applied to model one of the test to calibrate some of the parameters of the material model and to prove its effectiveness to represent the debonding of the adhesive layers. Finally, the numerical analyses of the tensile and compressive tests performed on the composite chiral components are presented and discussed.

\subsection{Basic aspects of the proposed approach}

To set up the previously described multilevel approach, numerical analyses performed at a refined level should prove capable to reliably model the response and the failure modes obtained in the experiments. Particularly, the chosen approach should represent the geometrical non-linearity exhibited by the composite chiral components, the stress levels experienced by plies in ligaments as well as the onset and propagation of damage in the adhesive layers between the ligaments.

To accomplish such objective a modeling technique based on the adoption of continuum shells connected by cohesive elements has been assessed and employed in non-linear quasi-static analyses carried out by means of the Abaqus Explicit code. In the chosen modeling approach, ligaments are modeled by 8-nodes continuum shell elements available in Abaqus, which implement conventional shell behavior but allow a direct discretisation of the surface of the laminates [16]. Such surfaces are connected by cohesive elements to represent the adhesive layers in the manufactured cells. Cohesive elements allows modeling the onset and propagation of damage at interfaces and have been already adopted to model interlaminar layers as well as bonded joints in composite structural elements [17-21].

Cohesive elements are often employed to model the behavior of idealized zero-thickness interfaces by means of traction-displacement laws. Such laws include a non-linear behavior, typically formulated by means of a continuum damage mechanics approach, suitable to model the strength as well as the toughness of the interfaces [17]. Toughness is modeled by controlling the energy required to completely degrade the interface in a non-linear analysis. In this work, as structural adhesive films have a not negligible thickness, they have not been modeled by means of a zero-thickness cohesive elements. Solid elements with reduced integration scheme (elements C3D8R [16]) have been employed and a cohesive law has been implemented in a user defined VUMAT subroutine to model their response.

Such elements connect the adjacent surfaces of two continuum shells, as shown in Fig. 19. The passages required to adapt the traction-displacement laws employed in cohesive elements into a stress-strain material model for solid elements are hereby briefly outlined. According to the adopted modeling technique, cohesive fracture processes within the adhesive layer is described by means of the relative displacement $\Delta=\mathrm{S}^{+}-\mathrm{S}^{-}$between the mid-point adjacent surfaces of the ligaments, sketched in Fig. 19a. A relative displacement in direction $z$ represents a fracture evolving in axial mode (mode $I$ ). The other components represent fractures evolving in shear modes, namely mode II or mode III. Such description of the fracture process is fundamental to model the toughness of the adhesive layer by means of links between the non-linear material model attributed to the cohesive elements and the critical energy release rates in the different fracture modes, $G_{I C}, G_{I I C}$ and $G_{I I I C}$.

Within the small strain assumption, the vector $\Delta$ can be expressed in terms of the average values of the out-of-plane strains acting in the material volume between the sublaminates. Such relation can be expressed as in Eq. (2), where th is the thickness of the adhesive layer.

$$
\begin{aligned}
& \{\varepsilon\}=\left\{\begin{array}{lll}
\varepsilon_{z z} & \gamma_{x z} & \gamma_{y z}
\end{array}\right\}^{T} \\
& \{\varepsilon\}=\{\Delta\} / t h
\end{aligned}
$$

When adjacent surfaces connected by a cohesive element move, a connection element, which is represented by a 8-noded brick with a reduced integration scheme, will experience the average strain state expressed in Eq. (2). If the in-plane response of the adhesive is neglected, the response of the solid element can be limited to the out-of-plane components, as in Eq. (3), where a scalar damage variable, $d$, is introduced to model material non-linearities.

\section{$\square$ Laminated continuum shell

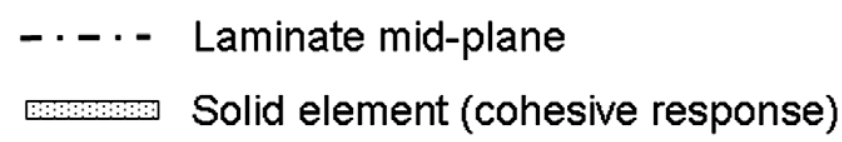

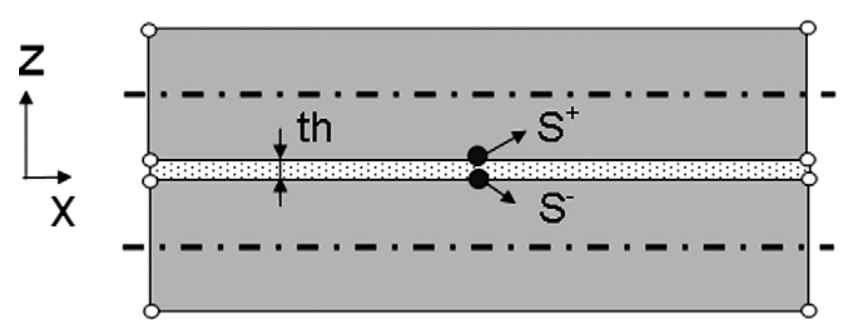

(a)

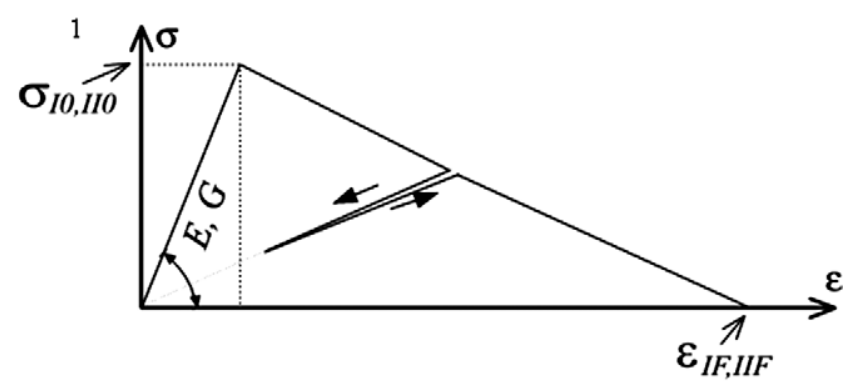

(b)

Fig. 19. Modeling technique for bonded laminates (a) and bi-linear response adopted in the cohesive material model (b) 


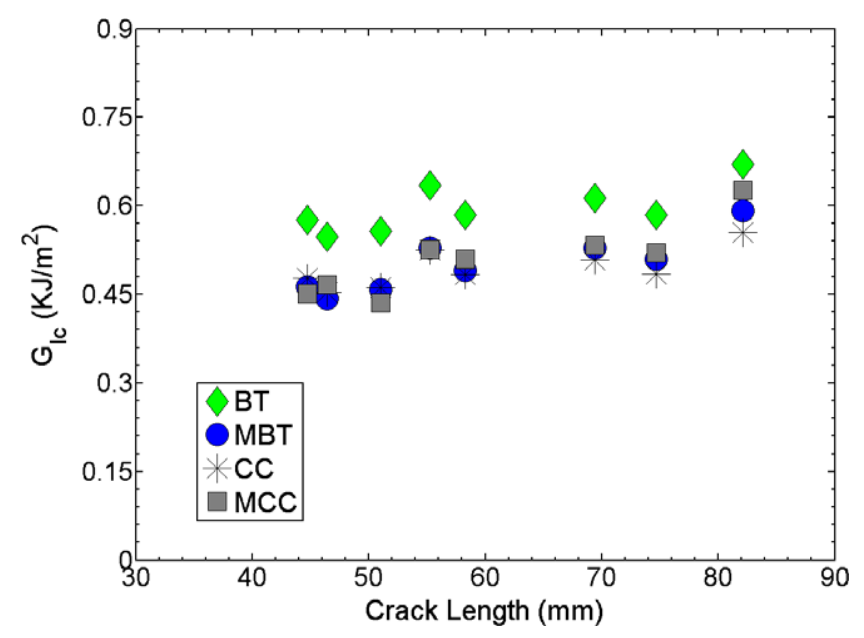

Fig. 20. $R-a$ curve evaluated by DCB test results on AF-163-2K adhesive.

$$
\left\{\begin{array}{l}
\sigma_{z z} \\
\sigma_{x z} \\
\sigma_{y z}
\end{array}\right\}=\left[\begin{array}{ccc}
E_{z z} & 0 & 0 \\
0 & G_{x z} & 0 \\
0 & 0 & G_{y z}
\end{array}\right](1-d)\left\{\begin{array}{l}
\varepsilon_{z z} \\
\gamma_{x z} \\
\gamma_{y z}
\end{array}\right\}
$$

On the basis of Eqs. (2) and (3), it is possible to formulate the aforementioned links between the non-linear material model and the critical energy release rates, which are reported in Eq. (4).

$$
\begin{aligned}
& \int_{0}^{\infty} \sigma_{z z} d \Delta_{I}=\operatorname{th} \int_{0}^{\infty} \sigma_{z z} d \varepsilon_{z z}=G_{I C} \\
& \int_{0}^{\infty} \sigma_{x z} d \Delta_{I I}=\operatorname{th} \int_{0}^{\infty} \sigma_{x z} d \gamma_{x z}=G_{I I C} \\
& \int_{0}^{\infty} \sigma_{y z} d \Delta_{I I I}=\operatorname{th} \int_{0}^{\infty} \sigma_{y z} d \gamma_{y z}=G_{I I I C}
\end{aligned}
$$

Eq. (4) states that the energy absorbed by the solid cohesive element during a deformation process must be limited. Moreover, such energy must correspond to the energy required to create a new free surface once that the element is completely degraded and the fracture process concluded.

The evolution of damage in the constitutive response that is reported in Eq. (3) must lead to a response that fulfils the links formulated in Eq. (4) and must also take into account mixed mode processes. The laws of damage evolution that are employed in this work are adapted from the ones devised in [17] for zero-thickness cohesive elements, which are based on bi-linear traction-displacement laws. In the material model, the parameters relevant to processes evolving in mode II and mode III are considered identical. Fig. 19b reports the chosen bi-linear approach that models the two basic fracture opening modes: the axial mode, driven by a $\sigma_{I}$ equivalent stress component, and the shear mode, driven by a $\sigma_{I I}$ equivalent stress component, with $\sigma_{I I}=\left[\left(\sigma_{x z}\right)^{2}+\left(\sigma_{y z}\right)^{2}\right]^{1 / 2}$.

The material model takes also into account the fundamentally different response of the interface to axial tension and compression. In fact, the equivalent stress driving damage in a mode-I fracture process, $\sigma_{I}$, turns out to be $\sigma_{z z}$ if $\sigma_{z z}>0$, but it is set to zero if $\sigma_{z z} \leqslant 0$. Moreover, the scalar damage variable introduced in Eq. (3) does never affect the material stiffness $E_{z z}$ if $\sigma_{z z} \leqslant 0$. Such behavior avoids the interpenetration of two adjacent surfaces even if the interfacial layer is completely degraded in the other directions. Mixed modes $I-I I$ processes are addressed by means of quadratic strength and toughness criteria as described in detail in [17].

The adopted law does not take into consideration the peculiar behavior of ductile adhesive in cohesive fracture mode $I$ processes. According to [18] such behavior can be described by means of a cohesive zone approach, but it is better represented by adopting a trapezoidal rather than a bi-linear response. The adopted law will be applied to model one of the test performed to characterize the adhesive toughness to show that it can be considered adequate to approximately model ligament debonding.

The choice of the explicit time integration scheme for the approach allows better handling with contact conditions and it is inherently capable to model sudden failures, characterized by unstable propagation of damages in the layers modeled by cohesive elements. However, the required computational times are noticeably increased with respect to implicit analyses [20].

\subsection{Characterization of adhesive toughness}

The cohesive material model of the adhesive layers requires the knowledge of the adhesive toughness in the axial and shear

(a)

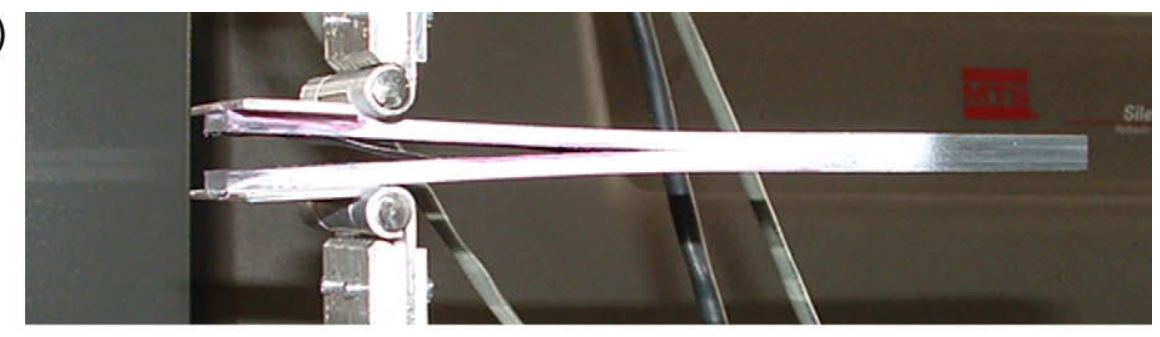

(b)

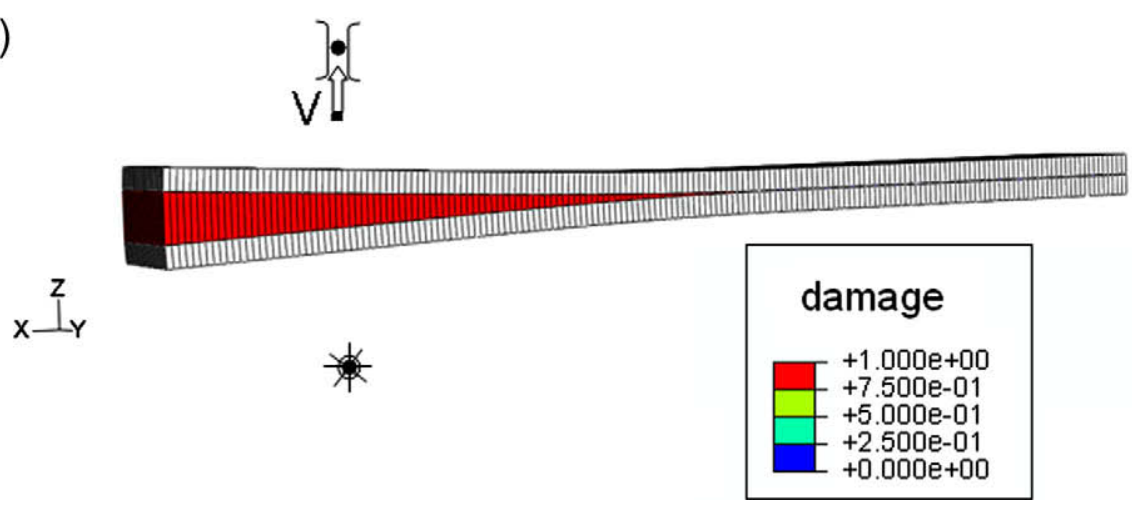

Fig. 21. DCB test (a) and numerical model (b) for the characterization of adhesive. 
Table 2

Material parameters of adhesive layers in numerical analyses.

\begin{tabular}{lll}
\hline & 1 st analysis & 2nd analysis \\
\hline$E_{z z}$ & $2.0 \mathrm{GPa}$ & $2.0 \mathrm{GPa}$ \\
$G_{X z}=G_{X z}$ & $0.75 \mathrm{GPa}$ & $0.75 \mathrm{GPa}$ \\
$\sigma_{I 0}$ & $30 \mathrm{MPa}$ & $30 \mathrm{MPa}$ \\
$\sigma_{I I 0}$ & $50 \mathrm{MPa}$ & $50 \mathrm{MPa}$ \\
$G_{I C}$ & $0.45 \mathrm{~kJ} / \mathrm{m}^{2}$ & $0.60 \mathrm{~kJ} / \mathrm{m}^{2}$ \\
$G_{I I C}$ & $0.67 \mathrm{~kJ} / \mathrm{m}^{2}$ & $0.67 \mathrm{~kJ} / \mathrm{m}^{2}$ \\
\hline
\end{tabular}

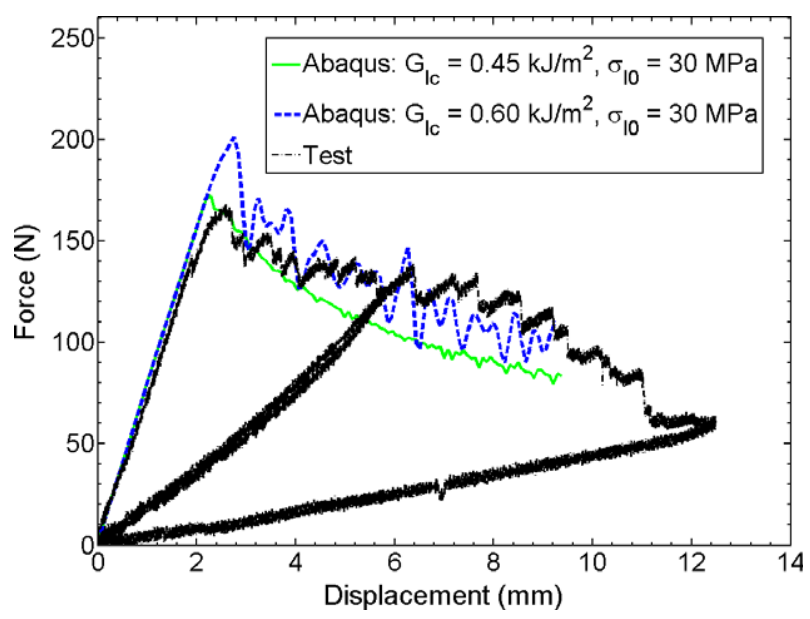

Fig. 22. Numerical-experimental correlation of DCB test.

fracture mode. Considering the tests performed on the chiral composite elements, the fracture process experienced by the adhesive in correspondence of tensile failure essentially occurred in mode I. The DCB (Double Cantilever Beam) test has been adopted as in [18] to evaluate $G_{I C}$ in a bonded joint using the same adhesive and composite fabric of the chiral cells. To complete the characterization of adhesive toughness, ENF (End Notched Flexure) tests [22] have also been carried on the bonded joints.

Specimens have been manufactured by bonding two laminates with a $[0]_{40}$ lay-up. The pre-cured laminates have been bonded by introducing a film of AF-163-2K adhesive, with the exclusion of a pre-cracked zone that has also been protected by a PTFE film. The bonded specimens have been cut to obtain eight $200 \mathrm{~mm} \times 25 \mathrm{~mm}$ specimens with a $[0]_{40}[\mathrm{AF}-163-2 \mathrm{~K}][0]_{40}$ layup and an average thickness of $7.8 \mathrm{~mm}$.

DCB test have been performed on four of the manufactured specimens. Pre-opening tests have been carried out in agreement with ASTM standard [23]. The four specimens have been subsequently subjected to a series of DCB opening tests, consisting of loading and unloading cycles. The crack advance has been monitored by means of dye penetrant techniques at the end of each cycle.

Fig. 20 presents the $R-a$ curve obtained in the tests by adopting a classic Beam Theory (BT) and the more reliable data reduction schemes suggested in [23] and also applied in [18] for ductile epoxy adhesive, namely Modified Beam Theory (MBT), Compliance Calibration (CC) and Modified Compliance Calibration (MCC). A critical energy release rate $G_{I C}$ bounded between $0.45 \mathrm{~kJ} / \mathrm{m}^{2}$ amd $0.60 \mathrm{~kJ} / \mathrm{m}^{2}$ has been evaluated from the experiments.

Four ENF tests have been carried out on the bonded specimens, after the pre-opening tests. The application of Beam Theory [22] to the ENF test data gave an average value $G_{I I C}$ of $0.670 \mathrm{~kJ} / \mathrm{m}^{2}$.

\subsection{Application of the numerical approach to DCB specimens}

A model of a DCB test has been developed and solved using Abaqus Explicit according to the previously presented numerical approach. Fig. 21 shows one of the performed DCB tests that started with an initial crack length of $46.4 \mathrm{~mm}$ and consisted of two loading-unloading phases, the first one until to a crack length of $69.4 \mathrm{~mm}$ and the second one until a final crack length of $119.6 \mathrm{~mm}$.

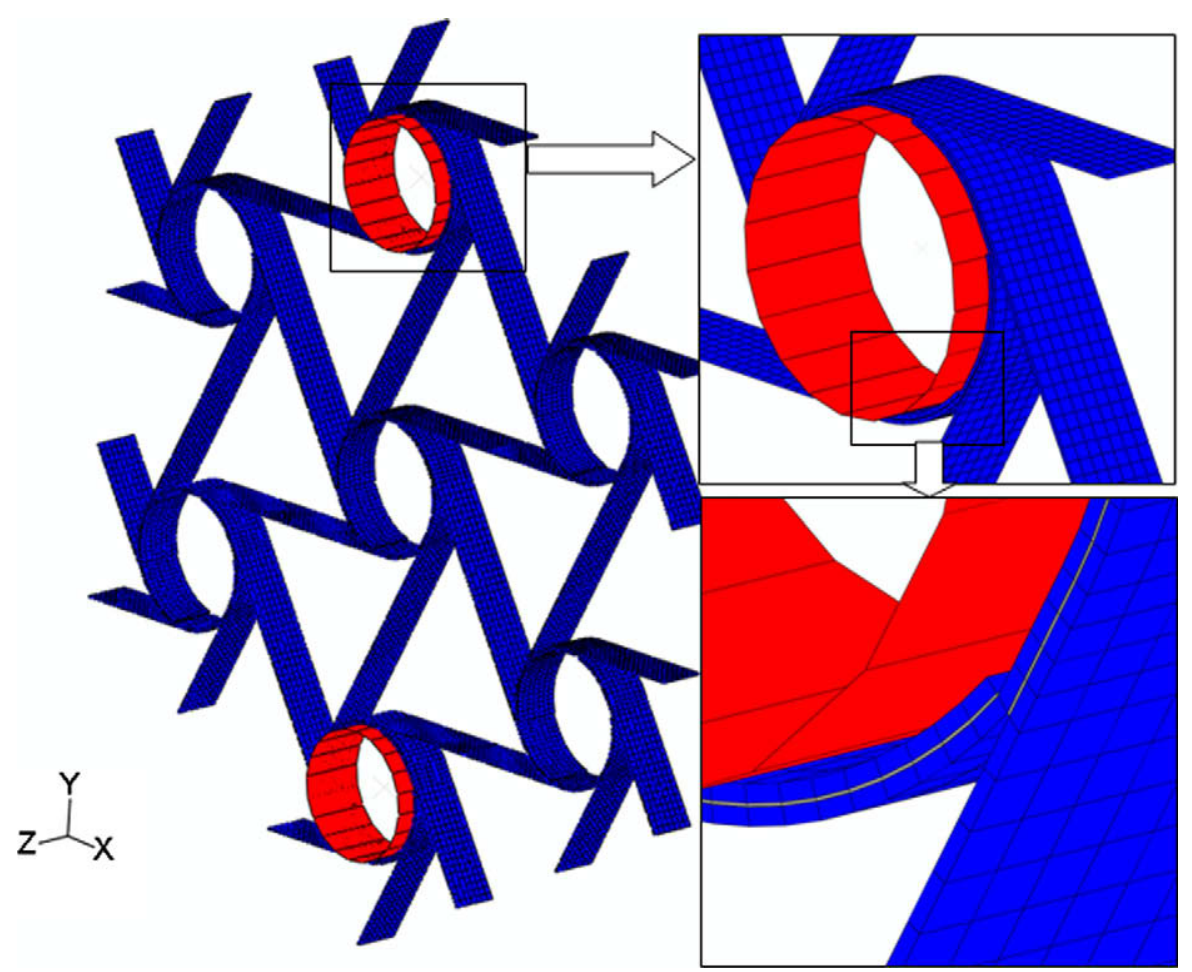

Fig. 23. Numerical model of the chiral composite component. 


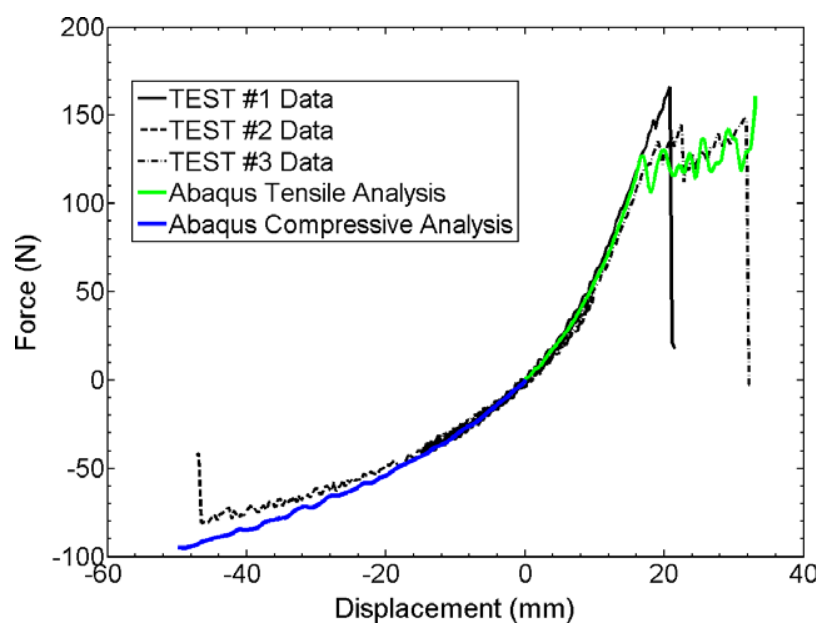

Fig. 24. Numerical-experimental correlation of the force vs. displacement responses for the chiral components.

Fig. 21b shows the model of the test consisting of two $[0]_{40}$ sublaminates, represented by the continuum shells, connected by the cohesive elements. An element size of $1.2 \mathrm{~mm}$ is adopted on the horizontal plane. Carbon fabric plies are characterized as an orthotropic elastic material with the characteristics reported in $\mathrm{Ta}$ ble 1 . Two analyses are hereby presented corresponding to two different choices of $G_{I C}$. The material parameters adopted in the two analyses for the cohesive material model are given in Table 2 . The identification of the $\sigma_{I 0}$ strength level (30 MPa) derives from an iterative process that also considers the results obtained by the numerical model of the chiral component, which will be presented in the following paragraph. The $\sigma_{I I 0}$ strength level has been set to a conservative value ( $50 \mathrm{MPa}$ ).

Boundary conditions are applied to two nodes belonging to rigid bodies that are connected to the surfaces of the modeled specimen. The contour presented in Fig. $21 \mathrm{~b}$ is referred to damage in the cohesive element.

Fig. 22 presents the obtained numerical-experimental correlation. The onset of crack propagation, corresponding to the load peak, is well captured by the numerical analysis with $G_{I C}=0.45 \mathrm{~kJ} / \mathrm{m}^{2}$. Increasing the toughness level to $0.60 \mathrm{~kJ} / \mathrm{m}^{2}$ allows a better correlation for large crack lengths, although the load corresponding to the initial propagation turns out to be overestimated. Such discrepancies are related to differences between the modeled bi-linear course and the actual response of the ductile adhesive layers. Overall, it can be observed that the behavior of the adhesive layer undergoing a mode $I$ cohesive fracture process is qualitatively well represented and that the model calibrated according to $G_{I C}=0.45 \mathrm{~kJ} / \mathrm{m}^{2}$ represents a reasonably conservative choice to predict the load required to propagate a mode-I fracture within the adhesive layer.

\subsection{Refined model of the chiral composite element}

A model of the chiral components which have been presented at Sections 3 and 4 has been developed considering half of the out-ofplane depth $(25 \mathrm{~mm})$ and applying symmetry constraints. The model is presented in Fig. 23. All ligaments are modeled by continuum shell elements with a $[0]_{5}$ lay-up of CC90/ET443 SEAL carbon

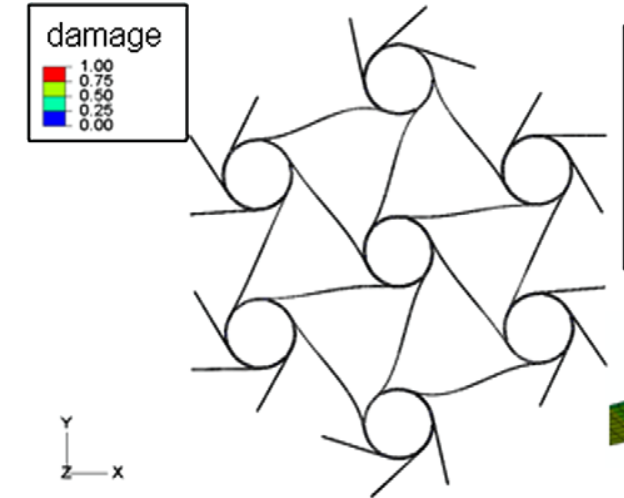

(a)

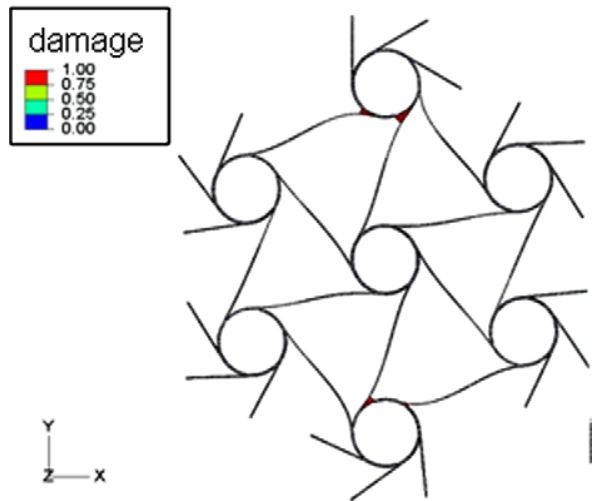

(c)

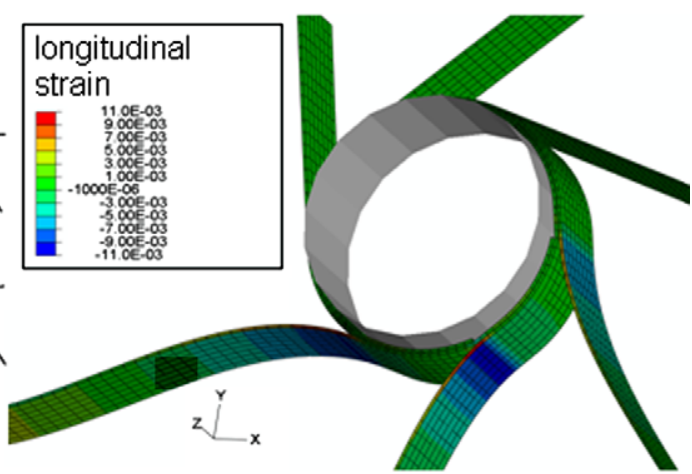

(b)

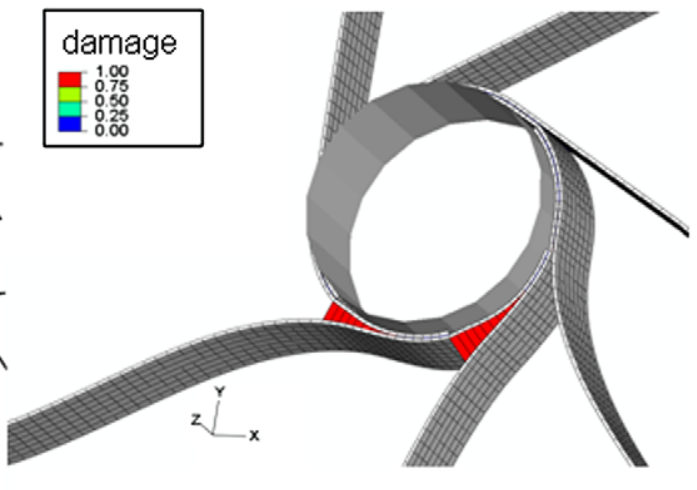

(d)

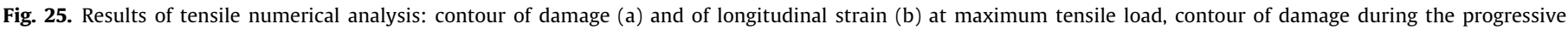
debonding of ligaments (c, d). 
fabric layers. The size of the elements on the plane of laminates varies from $1.2 \mathrm{~mm}$ to $2 \mathrm{~mm}$. Adhesive layers with a thickness of $0.1 \mathrm{~mm}$ are represented by cohesive elements characterized by the previously calibrated material model. Two analytical cylindrical surfaces model the constraints applied in the experiments and a contact interaction has been set between such rigid surfaces and the inner surfaces of the ligaments.

Material models are calibrated using $G_{I C}=0.45 \mathrm{~kJ} / \mathrm{m}^{2}$. The other properties have been set according to the values reported in Tables 1 and 2 . A tensile and a compressive analysis have been performed by imposing proper velocity boundary conditions to the moving cylinder. Different analyses have also been performed to identify the interlaminar strength $\sigma_{I 0}$, but for the sake of brevity, only the results obtained with the final set of material parameters will be presented.

The numerical force vs. displacement response, reported in Fig. 24 for the tensile as well as the compressive analyses is in good agreement with the experimental data.

As far as the tensile behavior is concerned, non-linearity in elastic range is well represented and the maximum load corresponds to the load levels recorded in TEST \#3. Fig. 25a is taken in correspondence of maximum load. It can be observed that the overall deformed shape is in agreement with the experimental evidence presented in Fig. 14c. Fig. 25b refers to the deformation at maximum load as well and presents the contour of the longitudinal strain along the ligaments close to one of the constrained nodes. The contour indicates that minimum strains in the range -0.011 $\div-0.009$ are reached on the compressed sides of the ligaments. Such results are in agreement with the strain gauge measures acquired during the tests and presented in Fig. 16. During the numerical analysis, at the maximum load, damage develops in the adhesive layers between the ligaments in correspondence of the constraints. Fig. 25c and d refer to the contour of damage in the adhesive layer during such post-failure phase. It can be concluded that the numerical load plateau exhibited by the numerical response reported in Fig. 24 corresponds to the progressive debonding of ligaments, as in TEST \#3. Fig. 25c also indicates that damage develops only at the constrained nodes of the component and the comparison with Fig. 14d shows that structure overall deformed shape during the debonding of ligaments is well represented by the numerical model. Comparison between Figs. 15 and $24 \mathrm{~d}$ also confirms that the numerical model can adequately describe the debonding mechanism during the tensile failure mode.

The numerical-experimental correlation of force vs. displacement response in the compressive analysis is reported in Fig. 24.

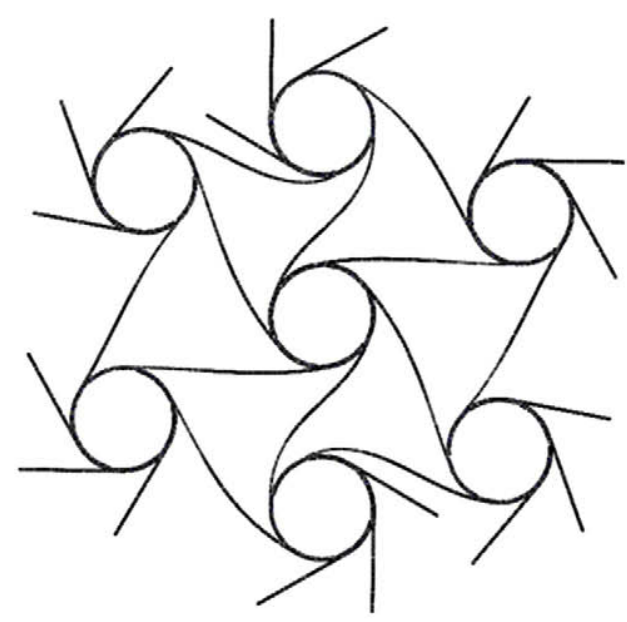

(a)

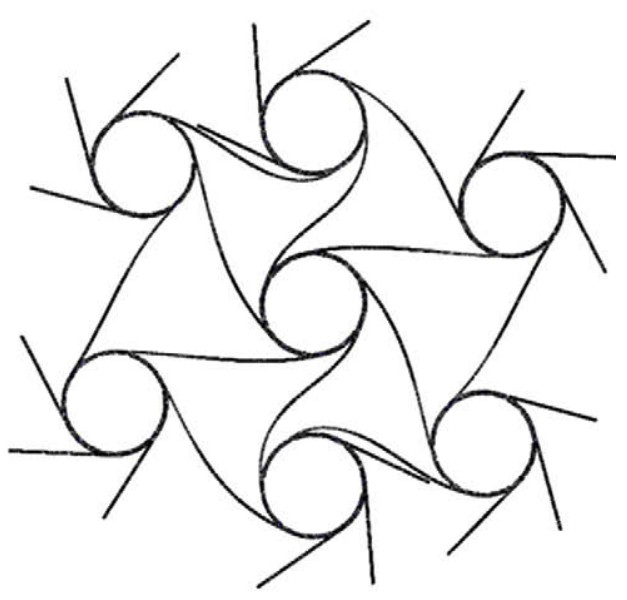

(b)

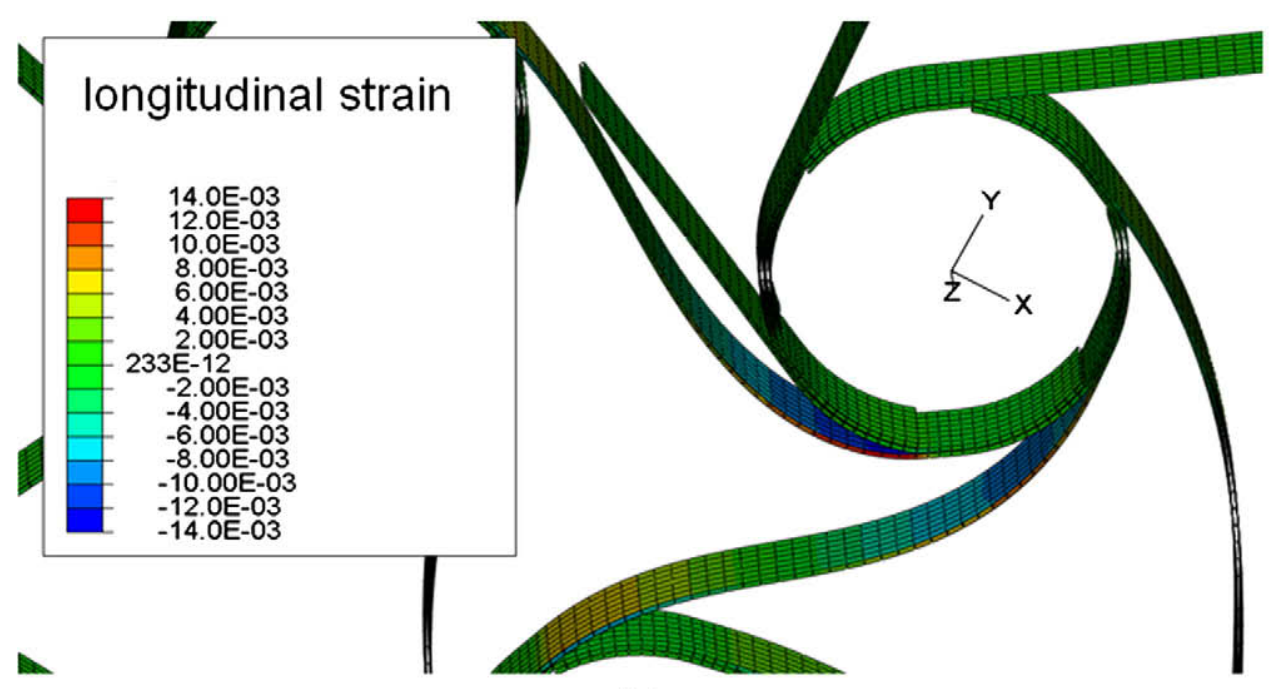

(c)

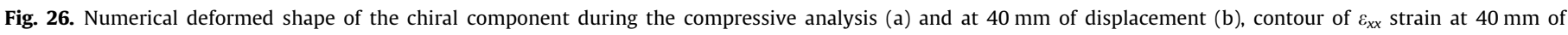
displacement (b).

Please cite this article in press as: Bettini P et al. Composite chiral structures for morphing airfoils: Numerical analyses and development of a manufacturing process. Composites: Part B (2009), doi:10.1016/j.compositesb.2009.10.005 
Non-linear behavior is well represented until a displacement of $-20 \mathrm{~mm}$. Thereafter, the numerical model turns out to be slightly stiffer than the component. Fig. 26a and b report the numerical deformed shapes obtained in the compressive analysis. Such figures can be compared with Fig. 17a and b. The contour of the numerical strains on the ligaments at about $40 \mathrm{~mm}$ of displacement, almost the end of the analyses, is reported in Fig. 26c. It indicates that strains of -0.014 are reached in the zone of maximum bending. Such contour is in agreement with the experimental evidence as far as the location of the critical points for ligament failure is concerned (see Fig. 17b and c). Strain levels during bending are also in good correlation with the acquired strain gauge signals reported in Fig. 18.

Hence, the developed numerical approach seems capable to model the response in the elastic range of the composite chiral component, as well as to predict the failure mode and the maximum loads carried by the structure.

\section{Conclusions}

The paper investigates the properties of a chiral honeycomb and proposes a manufacturing process using composite materials. The considered configuration finds potential application as part of a morphing airfoil, as described in the first part of the paper. Specifically, long fibers reinforced polymers can be exploited to enhance the morphing capability of chiral structural topologies. The technological process developed in the second part of this study represents a feasible and easy solution to experimentally investigate the properties of the considered class of composite honeycombs with non-conventional geometric characteristics. As far as the mechanical properties of the manufactured chiral components are concerned, the manufacturing approach has been applied in this paper with good results. The separate production of ligaments and their subsequent bonding allow producing elements that can work almost completely exploiting the in-plane strength of the composite plies adopted in this work.

Experimental results confirm the appealing properties of chiral honeycombs and illustrate their possible failure modes as well as their highly non-linear response resulting from geometrical effects at large displacements. Experiments considered an homogeneous lay-up of carbon fabric plies, but the adoption of other stack-up sequences, made of glass- or carbon-reinforced plies, can potentially improve the morphing performances of the structure in terms of maximum allowable displacement before the activation of failure mechanisms. Tests are also interpreted through a numerical approach characterized by the application of cohesive elements that model failure in the adhesive film. The approach accurately models the response and the failure of the tested elements, so that it could be adopted to identify the strength level of a composite chiral structure once the geometrical characteristics as well as the layup sequence of the unit cells have been selected.

Overall, the presented numerical and technological activities support the design and manufacture of chiral composite cores as effective means to provide aircraft structures with morphing capabilities. The developed approach and process allow the experimental and numerical investigation of design solutions for the skins and constraints of the chiral core, and the exploration of potential application of composite chiral structures in other engineering fields.

\section{Acknolwedgements}

This work is the result of a collaboration initiated by a visit of M. Ruzzene and A. Spadoni to the Dipartimento of Ingegneria Aerospaziale of Politecnico di Milano. The visit was partially supported by the project "Progetto Trasporto Aereo Regionale nel 2030" funded by Regione Lombardia. The activity at Politecnico di Milano was partially supported by FIRB project RBIP06AWF9 founded by Italian Ministry of Research.

\section{References}

[1] McGowan AR, Cox DE, Lazos BS, Waszak MR, Raney DL, Siochi EJ, et al. Biologically inspired technologies. In: NASA's morphing project, proceedings of SPIE smart structures and materials conference. San Diego, CA, March, 2003. p. $1-13$.

[2] Rodriguez AR. Morphing aircraft technology survey. In: Proceedings of 45th AIAA aerospace sciences meeting and exhibit. Reno, NV, January, 2007 [AIAA Paper 2007-1258].

[3] Bowman J, Sanders B, Weisshaar T. Identification of military morphing aircraft missions and morphing technology assessment. In: Proceedings of SPIE smart materials and structures conference. San Diego, CA March, 2002, p. 121-32.

[4] Chopra I. Review of state of art of smart structures and integrated systems. AIAA J 2002;40(11):2145-87.

[5] Lakes RS. Deformation mechanisms in negative Poisson's ratio materials: structural aspects. J Mater Sci 1991;26:2287-92.

[6] Prall D, Lakes RS. Properties of a chiral honeycomb with a Poisson's ratio of -1 . Int J Mech Sci 1997;39(3):305-14.

[7] Bornengo D, Scarpa F, Remillat C. Evaluation of hexagonal chiral structure for morphing airfoil concept. Proc Inst Mech Eng Part G J Aerospace Eng 2005;219(G3):185-92.

[8] Spadoni A, Ruzzene M. Numerical and experimental analysis of the static compliance of chiral truss-core airfoils. J Mech Mater Struct 2007;2(5): 965.

[9] Spadoni A, Ruzzene M. Static aeroelastic response of chiral truss-core airfoils. J Intell Mater Syst Struct 2007:5

[10] Martin J, Heyder-Bruckner JJ, Remillat C, Scarpa F, Potter K, Ruzzene M. The hexachiral prismatic wingbox concept. Phys Status Solidi B 2008;245(3):570-7.

[11] Spadoni A, Ruzzene M, Scarpa F. Dynamic response of chiral trus-core assemblies. J Intell Mater, Syst Struct 2006;17(11):941-52.

[12] ANSYSr. Theory Reference. ANSYS Academic Research, Release 11.0, 2007.

[13] Composite material handbook, vol. 2. Polymer matrix composite materials properties. MIL-HDBK-17-2E, 1999, Department of Defense, USA.

[14] Sala G. Advances in elastomeric tooling technology. Mater Des 1996;17(1): 33-42.

[15] Richards JM, Wostenholm GH, Yates B, Darby MI, Phillips LN. Thermal properties of carbon-filled silicone rubbers for use in thermal expansion moulding. J Mater Sci 1990;25:721-4.

[16] Abaqus. Analysis and User's Manual Version 6.7. Dassault System, 2007.

[17] Davila CG, Camanho PP, de Moura MSFS. Progressive analyses of skin/stringer debonding. In: Proceedings of American society for composites, 16th technical conference. Blacksburg, VA, September, 2001.

[18] De Moura MFSF, Campilho RDSG, Gonçalves JPM. Crack equivalent concept applied to the fracture characterization of bonded joints under pure mode I loading. Compos Sci Technol 2008;68:2224-30.

[19] Turon A, Davila CG, Camanho PP, Costa J. An engineering solution for mesh size effect in the simulation of delamination using cohesive zone models. Eng Fract Mech 2007;74:1665-82.

[20] Airoldi A, Sala G, Pasqualini F. Experimental and numerical investigation on the failure modes of thick composite laminates. In: Proceedings of 25th congress of aeronautical science, ICAS 2006, Hamburg, Germany, 2006, September.

[21] Zhou DW, Louca LA, Saunders M. Numerical simulation of sandwich T-joints under dynamic loading. Composites Part B 2008;39:973-85.

[22] Carlsson L, Gillespie JW. Mode-II interlaminar fracture of composites. In: Friedrich K, editor. Application of the fracture mechanics to composite materials. Amsterdam: Elsevier; 1989.

[23] American Society for Testing Materials. Standard test method for mixed mode I-mode II interlaminar fracture of unidirectional fiber reinforced polymer matrix composites. ASTM D 6671-01, 2001. 